\title{
Development and Evaluation of an Integral Sliding Mode Fault Tolerant Control Scheme on the RECONFIGURE Benchmark
}

\author{
L. Chen*and H. Alwi and C. Edwards \\ College of Engineering, Mathematics and Physical Sciences, University of Exeter, UK
}

\begin{abstract}
SUMMARY
This paper describes the development, application and evaluation of a linear parameter-varying integral sliding mode control allocation scheme to the RECONFIGURE benchmark model to deal with an actuator failure/fault scenario. The proposed scheme has the capability to maintain close to nominal (fault free) load factor control performance in the face of elevator failures/faults, by including a retro-fitted integral sliding mode term and then re-routing (via control allocation) the augmented control signal to healthy elevators without reconfiguring the baseline controller. In order to mitigate any chattering appearing in the elevator demands, the retro-fitted signal is based on a super-twisting sliding mode structure. This produces a control signal which is continuous and does not have the discontinuous switching nature of traditional sliding mode schemes. The scheme is evaluated using an industrial Functional Engineering Simulator developed as part of the RECONFIGURE project. Monte-Carlo campaign results are shown to demonstrate the performance of the proposed scheme.
\end{abstract}

KEY WORDS: Integral sliding mode; Fault tolerant control; Control allocation

\section{INTRODUCTION}

A challenge for upcoming and future aircraft is the extension of automatic Guidance Navigation and Control (GNC) functions to reduce pilot workload and to optimize aircraft performance. One of the ways this can be achieved is to facilitate the automated handling of abnormal events (i.e. sudden unexpected faults/failures or changes in flight parameters) [1, 2]. The aim of the EU-FP7 funded project RECONFIGURE (Reconfiguration of Control in Flight for Integral Global Upset Recovery) is to investigate and develop advanced GNC technologies to optimize the aircraft's status by automatically reconfiguring the aircraft to its optimal flight condition. Various specific scenarios have been identified by Airbus, and high fidelity benchmark simulations have been developed during the project for testing fault tolerant control strategies based on different paradigms. This paper addresses the problem of maintaining close to nominal load factor ${ }^{\dagger}$ control performance in the face of elevator faults and failures.

The topic of fault tolerant control (FTC) has been widely developed in the last decade $[4,5,6,7,8,9]$ and many different paradigms have subsequently been applied to the problem of fault

\footnotetext{
*Correspondence to: College of Engineering, Mathematics and Physical Sciences, University of Exeter, UK.

†The load factor is defined as the ratio of the lift of an aircraft to its weight [3].
} 
tolerant flight control [10,11, 12]. For instance, control allocation (CA) $[13,14,15,16]$, modular or physical approaches [17, 18,19], model predictive control [20, 21], backstepping/nonlinear dynamic inversion [22, 23, 24], set invariant methods [25], $\mathcal{H}_{\infty}$ approaches [4, 26] and extensions to linear parameter-varying (LPV) systems [27, 28, 29, 30, 31, 32] have investigated. In recent years adaptive controllers have been studied and particularly those in an $l_{1}$ framework have also seen a renaissance [33, 34, 35, 36, 37, 38]. Sliding mode approaches have also been studied [39, 40, 41] and tested - particularly in Europe [13]. These papers argue that actuator faults can be viewed as a class of matched uncertainty and that the inherent robustness properties of sliding mode control schemes can be exploited. In this paper a particular sliding mode methodology is applied - the so called Integral Sliding Mode (ISM) approach [42]. In this paper, a time varying scheduling parameter dependent sliding surface is proposed that guarantees sliding for all time.

The approach proposed in this paper is quite different from the developments presented in [13] where conventional sliding mode controllers were designed for specific uncertain linear time invariant (LTI) or LPV plants. The approaches in [13] follow a conventional sliding mode design protocol: a sliding surface is first selected so that the closed-loop motion when constrained to the surface yields appropriate performance; then a control law is designed to ensure sliding on the surface can be maintained even in the presence of faults and failures. In this paper the sliding mode scheme is retro-fitted to an existing closed-loop system preserving the original existing control structure (see Figure 1). The retro-fitted ISM scheme has been applied to one of the RECONFIGURE benchmark problems: specifically, an elevator failure/fault scenario, wherein one elevator, randomly selected from the four available elevators, is assumed to be stuck at its zero position. Fault tolerant load factor control (C-NZ) performance is then evaluated over a wide range of the flight conditions, and with various challenging pilot excitations, using an industrial Functional Engineering Simulator (FES) [1].

One of the novelties in this paper is that the LPV/ISM scheme is retro-fitted to the existing control law and architecture. In the absence of faults the augmented LPV/ISM structure together with the original controller behaves exactly as the existing (fault free) nominal baseline LPV controller architecture. The effect of the sliding mode augmentation only appears in the presence of faults/failures. The scheme is bespoke for the aerospace system considered, but the ideas can be appropriately applied to a reasonable wide class of over actuated nonlinear systems. It is based on the LPV model of the closed-loop system using the baseline LPV controller and extends the LTI based ideas from [13]. By incorporating a control allocation element the overall controller guarantees that sliding can be maintained from time zero [43, 44]. For the purpose of reducing the appearance of chattering in the elevator demands, the retro-fitted (continuous) control law is based on a super-twisting sliding mode structure [45, 42]. The other main novelty is the level of rigor with which the FTC scheme is tested. The performance is tested within an industrial Functional Evaluation Simulator, a tool developed by DEIMOS space [1], to evaluate the technology readiness levels of control schemes for possible implementation on the actual hardware. The RECONFIGURE FES includes a high fidelity nonlinear model of a civil commercial aircraft (incorporating both actuator and sensor dynamics and all the protection logic which forms part of Electrical Flight Control laws). The existing industry supplied controller is not modified but is augmented with the ISM elements and the control allocation functionality. Very promising results have been obtained 
from the proposed scheme which is shown to preserve close to nominal closed-loop performance in the face of significant faults.

The notation used in this paper is quite standard: in particular the $\mathcal{L}_{2}$ gain of an operator $\mathcal{H}: \phi \mapsto \xi$ is defined as $\|\mathcal{H}\|_{2}:=\sup \frac{\|\xi\|_{2}}{\|\phi\|_{2}}, \forall \phi \in \mathcal{L}_{2}, \phi \neq 0$.

\section{DESCRIPTION OF LPV/ISM SCHEME}

In this section, a retro-fitted LPV/ISM fault tolerant scheme is developed from a theoretical standpoint. The overall architecture of the scheme is given in Figure 1 where the terms in red represent the elements which will be added to the existing feedback control (shown in black).

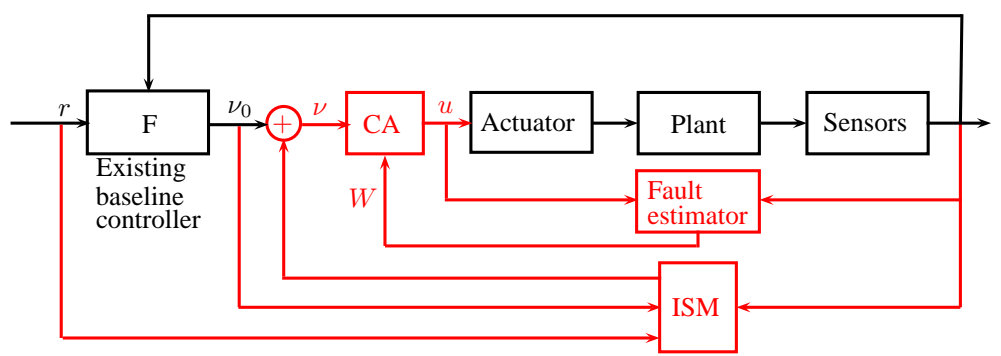

Figure 1. Overall linear parameter varying (LPV)/integral sliding mode (ISM) retrofitting structure

Consider a generic over actuated LPV system subject to actuator faults/failures modelled by

$$
\dot{x}(t)=A(\rho) x(t)+B(\rho) W(t) u(t)
$$

where the state vector $x \in \mathbb{R}^{n}$, the control input $u \in \mathbb{R}^{m}$, and the LPV scheduling parameter $\rho \in \Theta \subset \mathbb{R}^{q}$, where $\Theta$ is a compact bounded set. In this paper it is assumed $\rho$ is accurately measured. The plant matrix $A(\rho) \in \mathbb{R}^{n \times n}$ and the input matrix $B(\rho) \in \mathbb{R}^{n \times m}$. The diagonal weighting matrix

$$
W(t):=\operatorname{diag}\left(w_{1}(t), w_{2}(t), \ldots, w_{m}(t)\right)
$$

where the time varying scalars $w_{i} \in\left[\begin{array}{ll}0 & 1\end{array}\right]$ represent the effectiveness of the corresponding actuator [4]. For a fault-free actuator $w_{j}=1$, and for a completely failed actuator $w_{j}=0$. When $0<w_{j}<1$, the $j$ th actuator behaves with reduced effectiveness (i.e. it is faulty). Here it is assumed that the system has $l$ controlled outputs where $l<m$. Consequently the system is over actuated in the sense that more independent actuators are available for manipulation than are really necessary to independently control the outputs of interest. This redundancy will be exploited for fault tolerant control and offers the potential to retain performance in the face of total actuator failures.

\section{Assumption 2.1}

Assume the input distribution matrix $B(\rho)$ can be factorized as

$$
B(\rho)=B_{f} E(\rho)
$$


where $B_{f} \in \mathbb{R}^{n \times m}$ is a fixed matrix and $E(\rho) \in \mathbb{R}^{m \times m}$ is a matrix with varying components. It is further assumed $E(\rho)$ is invertible for all $\rho \in \Theta$.

This is a restriction and consequently a limitation on the class of system for which the results in this paper are applicable. Nevertheless it will be shown to be a reasonable one for the benchmark problem considered later in the paper. Next partition the fixed input distribution matrix component $B_{f}$ as

$$
B_{f}=\left[\begin{array}{l}
B_{f 1} \\
B_{f 2}
\end{array}\right]
$$

where $B_{f 1} \in \mathbb{R}^{(n-l) \times m}$ and $B_{f 2} \in \mathbb{R}^{l \times m}$. In (4) it is assumed that $\operatorname{rank}\left(B_{f 2}\right)=l$, i.e. full rank; and as in [46], that $\left\|B_{f 2}\right\| \gg\left\|B_{f 1}\right\|$. To achieve this structure, there may need to be a suitable permutation of the order of the states. An intuitive interpretation is that $B_{f}$ in the form of (4) is approximately rank $l$ since $B_{f 1} \simeq 0$. This implies that the control signals affect the plant predominately through the last $l$ channels in the states. If directly replicated actuators are present in (1) then this naturally contributes to rank deficiency in $B_{f}$ (since the columns of $B_{f}$ are not independent).

If the form in (4) can be achieved, then, without loss of generality, the last $l$ states can be scaled so that the property that $B_{f 2} B_{f 2}^{T}=I_{l}$ is satisfied (and hence $\left\|B_{f 2}\right\|=1$ ).

In this paper define a virtual control input according to

$$
v(t):=B_{f 2} E(\rho) u(t)
$$

where $v(t) \in \mathbb{R}^{l}$. The idea is then to design the virtual control law $v(t)$ using sliding mode ideas. For a given virtual control signal $v(t)$, the original physical control inputs $u(t) \in \mathbb{R}^{m}$ are defined as

$$
u(t):=E(\rho)^{-1} B_{f 2}^{T} v(t)
$$

\section{Remark 2.1}

Equation (6) represents a (fixed) control allocation structure. Based on the virtual control $v(t) \in \mathbb{R}^{l}$, equation (6) distributes/allocates this signal to the physical actuators through the control signal $u \in \mathbb{R}^{m}$ where $m>l$.

Note that the choice of $u(t)$ in (6) guarantees (5) is satisfied since $B_{f 2} B_{f 2}^{T}=I_{l}$. Substituting (4) and (6) into (1) yields

$$
\dot{x}(t)=A(\rho) x(t)+\underbrace{\left[\begin{array}{c}
B_{f 1} E(\rho) W(t) E(\rho)^{-1} B_{f 2}^{T} \\
B_{f 2} E(\rho) W(t) E(\rho)^{-1} B_{f 2}^{T}
\end{array}\right]}_{B_{w}(\rho)} v(t)
$$


Note that in (7) the system is driven by the virtual control input $v(t)$. In the fault-free case, $W(t)=I$ and (7) can be written in terms of the virtual control input as

$$
\dot{x}(t)=A(\rho) x(t)+\underbrace{\left[\begin{array}{c}
B_{f 1} B_{f 2}^{T} \\
I
\end{array}\right]}_{B_{v}} v(t)
$$

\section{Assumption 2.2}

Assume the pair $\left(A(\rho), B_{v}\right)$ is controllable for all $\rho \in \Theta$ and an existing state feedback control law $v(t)=-F(\rho) x(t)+L_{r}(\rho) r(t)$ has been designed to achieve appropriate tracking performance for the following closed-loop system

$$
\dot{x}(t)=\left(A(\rho)-B_{v} F(\rho)\right) x(t)+B_{v} L_{r}(\rho) r(t)
$$

for all $\rho \in \Theta$, where $r(t)$ is a reference signal for the controlled outputs.

Choose the (integral) sliding surface as

$$
\mathcal{S}:=\left\{x(t) \in \mathbb{R}^{n}: \sigma(x, \rho, t)=0\right\}
$$

where the switching function $\sigma(x, \rho, t)$ is defined as

$$
\sigma(x, \rho, t):=G x(t)-G x\left(t_{0}\right)-G \int_{0}^{t}\left(\left(A(\rho)-B_{v} F(\rho)\right) x(\tau)+B_{v} L_{r}(\rho) r(\tau)\right) d \tau
$$

and $G \in \mathbb{R}^{l \times n}$ represents the design freedom to be selected. The design freedom $G$ must be chosen to ensure the stability of sliding motion when the system in (1) is confined to the surface in (10). Suppose $G$ is chosen so that $G B_{v}=I_{l}$, i.e. it represents a right pseudo inverse of $B_{v}$. There is plenty of precedent for this choice: see for example [43]. Specifically here suppose

$$
G=\left[\begin{array}{ll}
0 & I_{l}
\end{array}\right]
$$

\section{Assumption 2.3}

In this paper it is assumed $W(t) \in \mathcal{W}_{\epsilon}$ where

$$
\mathcal{W}_{\epsilon}=\left\{W(t) \text { from (2) such that } B_{f 2} E(\rho) W(t) E(\rho)^{-1} B_{f 2}^{T}>\epsilon I_{l} \text { for all } \rho \in \Theta\right\}
$$

and $\epsilon$ is a small positive scalar.

\section{Remark 2.2}

This is a nonempty set since $W(t)=I \in \mathcal{W}_{\epsilon}$. The set $\mathcal{W}_{\epsilon}$ will define the faults which will be considered in the paper. Importantly, it includes the possibility of total failure. The fault model specified by (1), (3) and (13) is qualitatively similar to other such models encountered in the literature - for example [47]. The fact that in this paper $\operatorname{rank}(W(t))$ may be strictly less than $m$ means certain actuators can fail completely. The set (13) is more constrained than its equivalent condition in the linear case [48] (which would equate to the situation when $\epsilon=0$ ) because of the 
necessity of guaranteeing $\left\|E(\rho) W(t) E(\rho)^{-1} B_{f 2}^{T}\left(B_{f 2} E(\rho) W(t) E(\rho)^{-1} B_{f 2}^{T}\right)^{-1}\right\|$ remains bounded for all $W \in \mathcal{W}_{\epsilon}$.

In the analysis that follows, consider the operator $\mathcal{H}: \phi \mapsto \xi$ given by

$$
\mathcal{H}=\left\{\begin{array}{l}
\dot{z}=\left(A(\rho)-B_{v} F(\rho)\right) z+B_{o} \phi \quad z(0)=0 \\
\xi=F(\rho) z
\end{array}\right.
$$

where

$$
B_{o}=\left[\begin{array}{c}
B_{f 1} \\
0
\end{array}\right]
$$

Let $\gamma_{h}$ represent the $\mathcal{L}_{2}$ gain of the operator $\mathcal{H}$ (which is finite based on Assumption 2.1) and then define the worst case condition number $c=\max _{\rho \in \Theta}\|E(\rho)\|\left\|E^{-1}(\rho)\right\|$.

Theorem 2.1

Suppose $W(t)$ is available and $W(t) \in \mathcal{W}_{\epsilon}$. Then the sliding motion when the system is constrained to $\mathcal{S}$ in (10) is stable if

$$
\gamma_{h}\left(1+\frac{c}{\epsilon}\right)<1
$$

\section{Proof}

Using (7) and (11), the derivative of the switching function satisfies

$$
\dot{\sigma}=G B_{w}(\rho) v(t)+G B_{v} F(\rho) x(t)-G B_{v} L_{r}(\rho) r(t)
$$

where $B_{w}(\rho)$ is defined in (7). During sliding $\sigma=\dot{\sigma}=0$, and assuming the fault dependent gain $G B_{w}(\rho) \in \mathbb{R}^{l \times l}$ is nonsingular, the equivalent control signal [49] required to maintain sliding is

$$
v_{e q}=-\left(G B_{w}(\rho)\right)^{-1}\left(G B_{v} F(\rho) x(t)-G B_{v} L_{r}(\rho) r(t)\right)
$$

By direct computation

$$
G B_{w}(\rho)=B_{f 2} E(\rho) W(t) E(\rho)^{-1} B_{f 2}^{T}
$$

and therefore since by hypothesis $W(t) \in \mathcal{W}_{\epsilon}$ it follows

$$
\operatorname{det}\left(G B_{w}(\rho)\right) \neq 0
$$

and the equivalent control in (17) is well defined. Substituting (18) into (7) yields

$$
\dot{x}=A(\rho) x-B_{w}(\rho)\left(G B_{w}(\rho)\right)^{-1} G B_{v} F(\rho) x+B_{w}(\rho)\left(G B_{w}(\rho)\right)^{-1} G B_{v} L_{r}(\rho) r
$$

In the fault free case when $W(t)=I, B_{w}(\rho)=B_{v}$ and (21) is identical to the nominal system in (9). To analyse the general case when $W(t) \neq I$, adding and subtracting the term $B_{v} F(\rho) x$ to the right hand side of (21) yields

$$
\dot{x}=\left(A(\rho)-B_{v} F(\rho)\right) x+\left(B_{v}-B_{w}(\rho)\left(G B_{w}(\rho)\right)^{-1} G B_{v}\right) F(\rho) x+B_{w}(\rho)\left(G B_{w}(\rho)\right)^{-1} G B_{v} L_{r}(\rho) r
$$


Substituting for $G$ from (12), by direct computation

$$
B_{v}-B_{w}(\rho)\left(G B_{w}(\rho)\right)^{-1} G B_{v}=B_{o} \Phi(t)
$$

where $B_{o}$ is defined in (15) and

$$
\Phi(t):=B_{f 2}^{T}-E(\rho) W(t) E(\rho)^{-1} B_{f 2}^{T}\left(B_{f 2} E(\rho) W(t) E(\rho)^{-1} B_{f 2}^{T}\right)^{-1}
$$

Hence (when $r=0$ ) the differential equation (21) can be written as

$$
\dot{x}=\left(A(\rho)-B_{v} F(\rho)\right) x+B_{o} \Phi(\rho) F(\rho) x
$$

The stability of (25) can then be analysed using a small gain argument (Figure 2) based on the system in (14). Consider in Fig. 2, $\phi(t)=\Phi(t) \xi(t)$ where $\xi(t)=F(\rho) x(t)$ and the time varying gain $\Phi(t)$ is defined in (24), and the operator $\mathcal{H}$ is defined in (14):

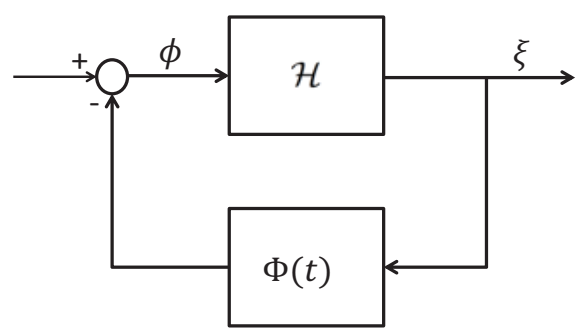

Figure 2. Small gain framework

If $W(t) \in \mathcal{W}_{\epsilon}$, then exploiting the fact that $\left\|B_{f 2}\right\|=1, \Phi(t)$ from (24) satisfies

$$
\|\Phi(t)\|<1+\frac{c}{\epsilon}
$$

where $\epsilon$ is defined in (13). Then if

$$
\gamma_{h}\left(1+\frac{c}{\epsilon}\right)<1
$$

using the small gain theorem, the system (25) is stable in an $\mathcal{L}_{2}$ sense.

\section{Remark 2.3}

Clearly the $\mathcal{L}_{2}$ gain of the operator $\mathcal{H}$ given in (14) depends on $B_{f 1}$ and $\gamma_{h} \rightarrow 0$ tends to zero as $\left\|B_{f 1}\right\| \rightarrow 0$. The $\mathcal{L}_{2}$ gain $\gamma_{h}$ is formally zero if $B_{f 1}=0$ (in which case the classic conditions for perfect control allocation are met [50]). In this situation (22) retains its nominal fault free performance provided sliding is maintained since $B_{o}=0$.

Next a (virtual) controller will be proposed to ensure sliding on $\mathcal{S}$ occurs in finite time and can be maintained.

Theorem 2.2

Assume $W(t)$ is known and $W(t) \in \mathcal{W}_{\epsilon}$. Consider the fixed control allocation law given by (6) where the virtual control law

$$
v(t)=\left(B_{f 2} E(\rho) W(t) E(\rho)^{-1} B_{f 2}^{T}\right)^{-1}\left(v_{s t}(t)-F(\rho) x(t)+L(\rho) r(t)\right)
$$


and

$$
\begin{aligned}
v_{s t}(t) & =-k_{1} \frac{\sigma(t)}{\|\sigma(t)\|^{1 / 2}}+z(t) \\
\dot{z}(t) & =-k_{2} \frac{\sigma(t)}{\|\sigma(t)\|}
\end{aligned}
$$

where $k_{1}$ and $k_{2}$ are two positive design scalars to be selected. Then a second order sliding mode can be induced in finite time on the surface $\mathcal{S}$ in (10).

\section{Proof}

It follows from (12) that $G B_{w}(\rho)=B_{f 2} E(\rho) W(t) E(\rho)^{-1} B_{f 2}^{T}$ and $G B_{v}=I$. Substituting (28) into the expression for $\dot{\sigma}$ in (17) yields the system

$$
\begin{aligned}
& \dot{\sigma}=-k_{1} \frac{\sigma(t)}{\|\sigma(t)\|^{1 / 2}}+z(t) \\
& \dot{z}=-k_{2} \frac{\sigma(t)}{\|\sigma(t)\|}
\end{aligned}
$$

Let $\zeta:=k_{1} \frac{\sigma(t)}{\|\sigma(t)\|^{1 / 2}}-z$ and define a Lyapunov candidate for system (30) as

$$
V(\sigma, z)=2 k_{2}\|\sigma\|+\frac{1}{2} z^{T} z+\frac{1}{2} \zeta^{T} \zeta
$$

where $V(\sigma, z)$ is everywhere continuous, and differentiable everywhere except on the surface $\mathcal{S}$ in (10). Differentiating (31) yields

$$
\dot{V}(\sigma, z)=\left(2 k_{2}+\frac{k_{1}^{2}}{2}\right) \frac{\sigma^{T} \dot{\sigma}}{\|\sigma\|}+2 z^{T} z-k_{1}\left(\frac{z^{T} \dot{\sigma}+\sigma^{T} \dot{z}}{\|\sigma\|^{1 / 2}}-\frac{1}{2} \frac{\left(z^{T} \sigma\right)\left(\sigma^{T} \dot{\sigma}\right)}{\|\sigma\|^{5 / 2}}\right)
$$

for $\sigma \neq 0$. Substituting (30) into (32) yields

$$
\begin{aligned}
\dot{V}(\sigma, z)= & -\left(2 k_{1} k_{2}+\frac{k_{1}^{3}}{2}\right) \frac{\sigma^{T} \sigma}{\|\sigma\|^{3 / 2}}+\frac{k_{1}^{2}}{2} \frac{\sigma^{T} z}{\|\sigma\|}+k_{1}^{2} \frac{z^{T} \sigma}{\|\sigma\|}-k_{1} \frac{z^{T} z}{\|\sigma\|^{1 / 2}}+k_{1} k_{2} \frac{\sigma^{T} \sigma}{\|\sigma\|^{3 / 2}} \\
& -\frac{k_{1}^{2}}{2} \frac{\left(z^{T} \sigma\right)\left(\sigma^{T} \sigma\right)}{\|\sigma\|^{3}}+\frac{k_{1}}{2} \frac{\left(z^{T} \sigma\right)\left(\sigma^{T} z\right)}{\|\sigma\|^{5 / 2}} \\
= & -\left(k_{1} k_{2}+\frac{k_{1}^{3}}{2}\right) \frac{\sigma^{T} \sigma}{\|\sigma\|^{3 / 2}}+k_{1}^{2} \frac{z^{T} \sigma}{\|\sigma\|}-k_{1} \frac{z^{T} z}{\|\sigma\|^{1 / 2}}+\frac{k_{1}}{2} \frac{\left(z^{T} \sigma\right)\left(\sigma^{T} z\right)}{\|\sigma\|^{5 / 2}}
\end{aligned}
$$

for all $\sigma$ not belonging to $\mathcal{S}$. Then it follows

$$
\dot{V}(\sigma, z)=-\left(k_{1} k_{2}+\frac{k_{1}^{3}}{2}\right)\|\sigma\|^{1 / 2}+k_{1}^{2} \frac{\left|\sigma^{T} z\right|}{\|\sigma\|}-k_{1} \frac{z^{T} z}{\|\sigma\|^{1 / 2}}+\frac{k_{1}}{2} \frac{\left|\sigma^{T} z\right|^{2}}{\|\sigma\|^{5 / 2}}
$$

Using the Cauchy-Schwarz inequality, it is easy to obtain

$$
\dot{V}(\sigma, z) \leq-\left(k_{1} k_{2}+\frac{k_{1}^{3}}{2}\right)\|\sigma\|^{1 / 2}+k_{1}^{2}\|z\|-\frac{k_{1}}{2} \frac{\|z\|^{2}}{\|\sigma\|^{1 / 2}}
$$


Define $\eta=\operatorname{col}\left(\|\sigma\|^{1 / 2},\|z\|\right)$ then (35) can be written as

$$
\dot{V}(\sigma, z) \leq-\frac{1}{\|\sigma\|^{1 / 2}} \eta^{T} \Omega \eta
$$

where

$$
\Omega=\left[\begin{array}{ll}
\Omega_{11} & \Omega_{12} \\
\Omega_{21} & \Omega_{22}
\end{array}\right]
$$

with elements

$$
\begin{aligned}
& \Omega_{11}=\frac{1}{2} k_{1}^{3}+k_{1} k_{2} \\
& \Omega_{12}=\Omega_{21}=-\frac{1}{2} k_{1}^{2} \\
& \Omega_{22}=\frac{k_{1}}{2}
\end{aligned}
$$

If $k_{1}>0$ and $k_{2}>0$, then $\Omega>0$ and $\dot{V} \leq-\frac{1}{\|\sigma\|^{1 / 2}} \lambda_{\min }(\Omega)\|\eta\|^{2}$. Also from (31), it follows $V=\eta^{T} P \eta \leq \lambda_{\max }(P)\|\eta\|^{2}$ where $P$ is an appropriate symmetric positive definite matrix. Then

$$
\dot{V} \leq-\gamma_{0} V^{1 / 2}
$$

holds for $\sigma \neq 0$ where $\gamma_{0}=\lambda_{\min }(\Omega) \sqrt{\lambda_{\min }(P)} / \lambda_{\max }(P)$. It follows $V(\sigma, z)$ is a continuous nonincreasing function. Then using the Lyapunov-like theorem for differential inclusions from [51], $\sigma$ and $\dot{\sigma}$ will converge to the origin in finite time and second order sliding is induced ${ }^{\ddagger}$.

\section{Remark 2.4}

In the representation in (1) there is no explicit inclusion of uncertainty, and consequently there is no analysis of uncertainty in Theorem 2.1 and in particular Theorem 2.2. However, it is well known that sliding mode schemes inherently provide robustness to matched uncertainty - i.e. uncertainties occurring in the input channels [42]. To address matched uncertainty, the gains $k_{1}$ and $k_{2}$ in Theorem 2.2 would need to satisfy additional constraints (beyond $k_{1}, k_{2}>0$ ). In particular $k_{2}$ would need to be chosen larger than the worst case upper bound on the rate of change of the uncertainty. For details see [52]. If the bounds on the uncertainty exist but are not known, it is also possible to incorporate an adaptive super-twisting structure $[54,55]$ to replace the structure in (29).

In the remainder of the paper, these ideas will be applied to the RECONFIGURE benchmark.

\section{DEVELOPMENT OF LPV MODEL OF THE RECONFIGURE BENCHMARK}

The RECONFIGURE benchmark is a nonlinear highly representative model of a generic Airbus civil aircraft. The benchmark contains a baseline gain-scheduled controller, detailed actuator and sensor models, as well as the angle of attack and speed protection components, and measurement filters. This aircraft model which has been developed within the EU funded RECONFIGURE

${ }^{\ddagger}$ For rigorous details of the Lyapunov analysis see [52, 53]. 
project is used as the basis for all the scenarios and for testing different fault detection and fault tolerant control strategies within the consortium [2, 1]. The simulation model of RECONFIGURE is 'invisible' for design purposes, and runs in a LINUX environment. The 'flight control computer' component is extracted in a Simulink model which includes the baseline LPV controller, and ways to 'plug in' new designs [2]. Simplified LPV models are also available for the design of fault detection and isolation (FDI) and FTC schemes [2, 1].

In this paper an actuator (elevator) fault/failure scenario is considered. The actuator fault/failure, potentially caused by a faulty electronic component, a mechanical breakage or an erroneous flight control computer computation, is assumed to occur and lead to the loss of the corresponding control surface. The objective is to maintain the longitudinal load factor (C-NZ) performance in the face of actuator faults/failures in various operational conditions and during specific validation activities. In the RECONFIGURE benchmark actuator scenario, one elevator, randomly selected from the four elevators, is assumed to be stuck at its zero position (system reconfiguration) from the beginning of the simulation. To retain nominal C-NZ performance in the presence of actuator faults/failures, the proposed ISM/CA scheme will redistribute the virtual control signals to the remaining three healthy elevators.

In order to develop an LPV model to be used as a basis for ISM design, consider initially an LPV model of the longitudinal aircraft rigid-body dynamics

$$
\begin{aligned}
& \dot{x}_{p}=A_{p}(\rho) x_{p}+B_{p}(\rho) u_{p} \\
& y_{p}=C_{p}(\rho) x_{p}+D_{p}(\rho) u_{p}
\end{aligned}
$$

In (40) the system inputs are $u_{p}=\left[\begin{array}{ll}\delta_{e} & \delta_{s}\end{array}\right]^{T}$ i.e. an aggregated elevator signal (deg) (from the four individual separate surfaces) and the horizontal stabilizer control surface deflection (deg). The controlled outputs $y_{p}=\left[\begin{array}{ll}n_{z, \text { irs }} & q_{g y r}\end{array}\right]^{T}$ represent the load factor $(\mathrm{g})$ and pitch rate signals (deg/s). In (40), the system states are $x_{p}=\left[\begin{array}{lllll}q_{g y r} & V_{g} & \alpha & \theta & z_{g}\end{array}\right]^{T}$, which represent pitch rate (deg/s), ground velocity (kts), angle of attack (deg), pitch angle (deg) and geographical altitude (ft). The LPV variable $\rho \in \Theta \subseteq \mathbb{R}^{4}$ has components $\rho=\left[\begin{array}{llll}w & x_{c g} & V_{c} & z_{g}\end{array}\right]^{T}$ which represent mass (tons), $\mathrm{x}$-position of the centre of gravity (\%), calibrated airspeed (kts) and aircraft altitude (ft).

The matrices $A_{p}(\rho) \in \mathbb{R}^{5 \times 5}, B_{p}(\rho) \in \mathbb{R}^{5 \times 2}, C_{p}(\rho) \in \mathbb{R}^{2 \times 5}$ and $D_{p}(\rho) \in \mathbb{R}^{2 \times 2}$ are affine LPV matrices, in particular:

$$
A_{p}(\rho)=A_{0}+A_{1} w+A_{2} x_{c g}+A_{3} V_{c}+A_{4} z_{g}
$$

The LPV model in (40)-(41) has been built using multivariable polynomial least squares interpolation of the set of linear time-invariant models provided by Airbus. It can be shown this model is able to approximate the aircraft dynamics well in cruise conditions, however, in many of the more aggressive pilot excitations in the RECONFIGURE fault scenario, significant plantmodel mismatches exist. In this paper, the plant-model mismatch will be dealt with by ensuring the occurrence of a sliding mode [42] and by exploiting the robustness properties of the associated sliding motion. 
Here as in [56], the elevator dynamics are modelled as

$$
\begin{aligned}
\dot{x}_{a c t} & =-\underbrace{K_{e}\left(\rho, x_{a c t}, \dot{x}_{a c t}\right)}_{K_{e}(\cdot)} x_{a c t}+K_{e}\left(\rho, x_{a c t}, \dot{x}_{a c t}\right) \delta_{a} \\
\delta_{e} & =x_{a c t}
\end{aligned}
$$

where the scalar $\delta_{e}$ is the first component of $u_{p}$ in (40), and $\delta_{a}$ denotes the aggregated pitch command. As in [56]

$$
K_{e}(\cdot)=C_{0}(\rho)+C_{1}(\rho) \operatorname{sign}\left(\dot{x}_{a c t}\right)\left(x_{a c t}+C_{2}(\rho)\right)
$$

In the benchmark, it is filtered versions of the load factor and pitch rate which are available. To this end let $y_{f}=\left[\begin{array}{ll}n_{z f} & q_{f}\end{array}\right]^{T}$ represent the filtered load factor and pitch rate measurements. In terms of modelling the closed-loop fault free situation, the inclusion of these filter dynamics improves the level of fidelity. Here

$$
y_{f}=G_{f}(s) y_{p}
$$

where the transfer function matrix $G_{f}(s)$ is given by

$$
G_{f}(s)=G_{h q}(s) G_{n 2}(s)\left[\begin{array}{cc}
G_{n 1}(s) & 0 \\
G_{n 1}(s) G_{w o}(s) & G_{g d}(s)
\end{array}\right]
$$

where $G_{n 1}(s), G_{n 2}(s)$ are both notch filters, and $G_{w o}(s), G_{h q}(s)$ and $G_{g d}(s)$ represent washout filters, a low pass filter and the gyro filter delay, respectively. The filter structure in (45) is bespoke to the particular aircraft under study and comes from an understanding of the benchmark. Specific details about $G_{f}(s)$ cannot be given in this paper due to industrial restrictions.

Let the state space representation of $G_{f}(s)$ be given by

$$
\begin{aligned}
\dot{x}_{f} & =A_{l p} x_{f}+B_{l p} y_{p} \\
y_{f} & =C_{l p} x_{f}
\end{aligned}
$$

where $A_{l p} \in \mathbb{R}^{12 \times 12}, B_{l p} \in \mathbb{R}^{12 \times 2}$ and $C_{l p} \in \mathbb{R}^{2 \times 12}$. In particular, it can be verified that this system is minimum phase. It is important to model the dynamics of $G_{f}(s)$ since $n_{z f}$ is a function of $n_{z, \text { irs }}$ and $\dot{q}_{g y r}$ (i.e. $q_{g y r}$ filtered by $G_{w o}(s)$ ). Another benefit of taking into account $G_{f}(s)$ is the semiproper open-loop LPV system from (40) is transformed into a strictly-proper one. This significantly decreases the LPV-based design complexity.

Let the matrices $B_{p}(\rho)$ and $D_{p}(\rho)$ from (40) be partitioned as $B_{p}(\rho)=\left[\begin{array}{ll}B_{p 1}(\rho) & B_{p 2}(\rho)\end{array}\right]$ and $D_{p}(\rho)=\left[\begin{array}{ll}D_{p 1}(\rho) & D_{p 2}(\rho)\end{array}\right]$. In this partitioning process, the vectors $B_{p 1} \in \mathbb{R}^{5}$ and $D_{p 1} \in \mathbb{R}^{5}$ are distribution matrices corresponding to the aggregated elevator surface deflections whilst $B_{p 2}$ and $D_{p 2}$ correspond to the stabilizer control surface deflection. 
Combining the aircraft rigid body model, the actuator model and the filters yields an augmented (strictly proper) state-space model

$$
\begin{aligned}
& \underbrace{\left[\begin{array}{c}
\dot{x}_{f} \\
\dot{x}_{p} \\
\dot{x}_{a c t}
\end{array}\right]}_{\dot{x}_{a}}=\underbrace{\left[\begin{array}{ccc}
A_{l p} & B_{l p} C_{p}(\rho) & B_{l p} D_{p 1}(\rho) \\
0 & A_{p}(\rho) & B_{p 1}(\rho) \\
0 & 0 & -K_{e}(\cdot)
\end{array}\right]}_{A_{a}(\rho)} \underbrace{\left[\begin{array}{c}
x_{f} \\
x_{p} \\
x_{a c t}
\end{array}\right]}_{x_{a}}+\underbrace{\left[\begin{array}{cc}
0 & B_{l p} D_{p 2}(\rho) \\
0 & B_{p 2}(\rho) \\
K_{e}(\cdot) & 0
\end{array}\right]}_{B_{a}(\rho)} \underbrace{\left[\begin{array}{c}
\delta_{a} \\
\delta_{s}
\end{array}\right]}_{u_{0}} \\
& y_{f}=\underbrace{\left[\begin{array}{lll}
C_{l p} & 0 & 0
\end{array}\right]}_{C_{a}}\left[\begin{array}{c}
x_{f} \\
x_{p} \\
x_{a c t}
\end{array}\right]
\end{aligned}
$$

where $A_{a}(\rho) \in \mathbb{R}^{18 \times 18}, B_{a}(\rho) \in \mathbb{R}^{18 \times 2}$ and $C_{a} \in \mathbb{R}^{2 \times 18}$. In (47), the augmented states are given by $x_{a}=\left[x_{f}^{T}, x_{p}^{T}, x_{a c t}^{T}\right]^{T}$, where $x_{p}$ is from (40), $x_{a c t}$ is from (42), $x_{f}$ represents the filtered states from the representation in (44) and $u_{0}$ represents the manipulated inputs used to achieve ideal closed-loop system performance.

The pitch command in (42) is modelled by

$$
\delta_{a}=G_{d e l}(s) \delta_{c}
$$

where the LTI system $G_{d e l}(s)$ represents an approximation of the time delay within the actuator, the flight control unit and the pitch-order selection components. The signal $\delta_{c}(t)$ represents the existing gain scheduled scalar proportional-integral (PI) control law

$$
\delta_{c}(t)=\mathcal{K}\left(t, n_{z f}(t), r(t), n_{z f}\left(t-t_{d e l}\right), q_{f}(t), n_{z, \text { equi }}\right)
$$

where $t_{d e l}$ represents a transport delay. The control law in (49) is pre-specified (by Airbus in this case) and is not part of the design process.

For the ISM design which will be described in the next section all these parameters and transfer functions are assumed to be known. Define a new state $e_{I}$ representing the load factor tracking performance according to

$$
e_{I}=\int_{0}^{t}\left(n_{z f}(\tau)+n_{z, \text { equi }}-r(\tau)\right) d \tau
$$

where $r(t)$ represents the load factor command and $n_{z, \text { equi }}$ is the fixed load factor trim value. Decompose the output distribution matrix from (47) as

$$
C_{a}=\left[\begin{array}{l}
C_{1} \\
C_{2}
\end{array}\right]
$$

where $C_{1} \in \mathbb{R}^{1 \times 18}$ and $C_{2} \in \mathbb{R}^{1 \times 18}$ then combining (50) and (47) yields the following augmented LPV system

$$
\left[\begin{array}{c}
\dot{e}_{I} \\
\dot{x}_{a}
\end{array}\right]=\left[\begin{array}{cc}
0 & C_{1} \\
0 & A_{a}(\rho)
\end{array}\right]\left[\begin{array}{c}
e_{I} \\
x_{a}
\end{array}\right]+\left[\begin{array}{c}
0 \\
B_{a}(\rho)
\end{array}\right] u_{0}-\left[\begin{array}{l}
1 \\
0
\end{array}\right]\left(r-n_{z, \text { equi }}\right)
$$


In this paper, the aim of the proposed LPV/ISM scheme is to recover the nominal load factor performance (i.e. $C-N Z$ performance) in the face of elevator faults/failures. Since, in operational practice, the stabilizer is only used to maintain trim and provide longitudinal static stability, only the elevator signal will be manipulated through the retrofitted ISM scheme. The overall structure of the retro-fitted scheme for solving the RECONFIGURE benchmark problem is given in Fig. 3 where the terms shown in red represent the new elements added to the existing structure.

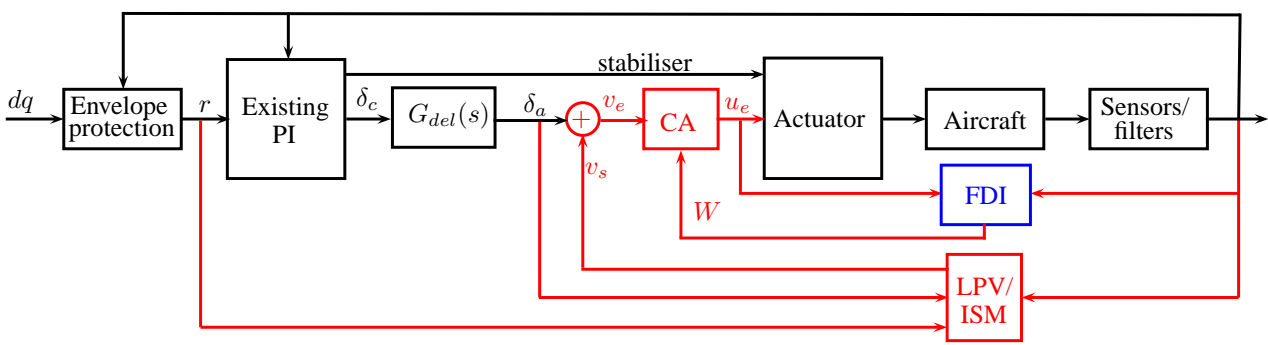

Figure 3. Overall LPV/ISM retro-fitting structure for RECONFIGURE benchmark problem. CA, control allocation; FDI, fault detection and isolation; PI, proportional-integral controller.

Let $B_{a}(\rho)$ in (47) be decomposed as

$$
B_{a}(\rho)=\left[B_{e}(\rho) \quad B_{s}(\rho)\right]
$$

Using the fact that $\delta_{s}=K_{s}(\rho)\left[\begin{array}{cc}e_{I}^{T} & x_{a}^{T}\end{array}\right]^{T}$ where $K_{s}(\rho)$ represents the gain scheduled feedback control law associated with the stabilizer, the LPV system in (52) can be written as

$$
\underbrace{\left[\begin{array}{c}
\dot{e}_{I} \\
\dot{x}_{a}
\end{array}\right]}_{\dot{x}}=\underbrace{\left.\left[\begin{array}{cc}
0 & C_{1} \\
0 & A_{a}(\rho)
\end{array}\right]+\left[\begin{array}{c}
0 \\
B_{s}(\rho)
\end{array}\right] K_{s}(\rho)\right)}_{A(\rho)} \underbrace{\left[\begin{array}{c}
e_{I} \\
x_{a}
\end{array}\right]}_{x}+\underbrace{\left[\begin{array}{c}
0 \\
B_{e}(\rho)
\end{array}\right]}_{\bar{B}_{e}(\rho)} \delta_{a}-\underbrace{\left[\begin{array}{c}
1 \\
0
\end{array}\right]}_{B_{r}}\left(r-n_{z, e q u i}\right)
$$

where $A(\rho) \in \mathbb{R}^{19 \times 19}, \bar{B}_{e}(\rho) \in \mathbb{R}^{19}$ and $B_{r} \in \mathbb{R}^{19}$.

In the analysis which follows, the original scalar control law signal $\delta_{a}$ from the existing controller will be re-allocated to the four individual elevators, in order to deal with faults and failures. Here, the original signal $\delta_{a}$ is split into four signals using

$$
\delta_{a}=B_{2} u_{e}
$$

where

$$
B_{2}:=\left[\begin{array}{llll}
1 & 1 & 1 & 1
\end{array}\right]
$$

and $u_{e} \in \mathbb{R}^{4}$ represents the four individual elevator commands. In (57) the structure of $B_{2}$ follows from the fact that $\delta_{a}$ in (47) (the aggregated elevator component) is the sum of the individual elevator components $u_{1}, \ldots, u_{4}$ from $u_{e}(t)$. 
Therefore, the LPV system in (54), taking into account actuator faults/failures and uncertainties/disturbances, can be written

$$
\dot{x}(t)=A(\rho) x(t)+\underbrace{\bar{B}_{e}(\rho) B_{2}}_{B(\rho)} W(t) u_{e}(t)+B_{r}\left(r(t)-n_{z, \text { equi }}\right)
$$

where $B(\rho) \in \mathbb{R}^{19 \times 4}$. In (57), $W(t) \in \mathcal{W}_{\epsilon} \subseteq \mathbb{R}^{4 \times 4}$ represents a diagonal semi-positive definite weighting matrix. The diagonal elements $w_{1}(t), w_{2}(t), w_{3}(t)$ and $w_{4}(t)$ of $W(t)$ represent the efficiency levels of the corresponding individual elevators as explained in Section 2. Comparing $B(\rho)$ in (57) with the generic case in equation (3) it follows that

$$
B(\rho)=\underbrace{\left[\begin{array}{c}
B_{f 1} \\
B_{f 2}
\end{array}\right]}_{B_{f}} \underbrace{2 K_{e}(\cdot) I_{4}}_{E(\rho)}
$$

where $B_{f 1}=0, K_{e}(\cdot)$ is from (42) and

$$
B_{f 2}=\left[\begin{array}{llll}
1 / 2 & 1 / 2 & 1 / 2 & 1 / 2
\end{array}\right]
$$

As in (5), the 'virtual' elevator input can be defined as

$$
v_{e}:=2 B_{f 2} K_{e}(\cdot) I_{4} u_{e}
$$

and then using the fact that $B_{f 1}=0$ and $B_{f 2} B_{f 2}^{T}=1$, the term $B(\rho) W u_{e}$ in (57), when $W=I_{4}$, becomes

$$
B(\rho) u_{e}=\left[\begin{array}{l}
B_{f 1} \\
B_{f 2}
\end{array}\right] K_{e}(\cdot) I_{4} K_{e}(\cdot)^{-1} B_{f 2}^{T} v_{e}=\underbrace{\left[\begin{array}{l}
0 \\
1
\end{array}\right]}_{B_{e}} v_{e}
$$

This model will form the basis of the ISM design described in the next section. It represents both a good approximation to the real nonlinear system, whilst also meeting the requirements necessary to apply the generic theory proposed in Section 2.

\section{DESIGN AND EVALUATION RESULTS}

This section describes the physical sliding mode control law to be implemented in the FES as well as the industrial constraints, testing activities and flight conditions to be considered for validation purposes. The design and FES Monte-Carlo campaign results are also shown in this section.

\subsection{Control Law Design}

For the aircraft system in (57)-(59), the integral sliding surface is chosen as

$$
\mathcal{S}:=\left\{x \in \mathbb{R}^{19}: \sigma(t, x, \rho)=0\right\}
$$


where the switching function $\sigma(t, x, \rho)$ is defined as

$$
\sigma(\cdot)=G(x(t)-x(0))-G \int_{0}^{t}\left(A(\rho) x(\tau)+B_{e} v_{0 e}(\tau)+B_{r}\left(r(\tau)-n_{z, e q u i}\right)\right) d \tau
$$

In (63), $B_{e}$ is defined in (61) and the nominal (virtual) control $v_{0 e}$ is defined by

$$
v_{0 e}:=K_{e}(\cdot) \delta_{a}
$$

where $\delta_{a}$ is defined in (48) and the row vector

$$
G=\left[\begin{array}{ll}
01 \times 18 & 1
\end{array}\right]
$$

Using the definition of $G$ in (65) it is easy to verify $G B_{e}=1$ and $G B_{r}=0$. In the case of actuator faults/failures, i.e. when $W(t) \neq I$, it follows from (63) and (57) that the derivative of $\sigma(\cdot)$ can be written as

$$
\dot{\sigma}(\cdot)=\underbrace{B_{f 2} W(t) B_{f 2}^{T}}_{\Psi(\cdot)} v_{e}-v_{0 e}
$$

where $v_{e}$ is defined in (60). In (66), $\Psi(\cdot) \neq 0$ is identical to the condition $B_{f 2} W(t) B_{f 2}^{T} \neq 0$. Using $B_{f 2}$ defined in (59), it is easy to verify

$$
\Psi(\cdot)=B_{f 2} W(t) B_{f 2}^{T}=\sum_{i=1}^{4} w_{i}(t)
$$

Since the $w_{i}(t) \geq 0$, provided at least one $w_{i}(t) \neq 0$ for $i=1, \ldots, 4$, the condition $\Psi(\cdot) \neq 0$ is satisfied.

\section{Remark 4.1}

Note comparing (58) with the general structure in (4) it follows in this example $B_{f 1}=0$. From Theorem 2.1, the nominal performance is retained and the 'small gain theorem' condition is automatically satisfied (and therefore the sliding surface can be established to be stable in the presence of faults/failures).

According to Theorem 2.2, the physical control law is given by

$$
\begin{aligned}
& u_{e}=B_{f 2}^{T} \Psi(\cdot)^{-1}(\delta_{a}-\underbrace{k_{1} K_{e}(\cdot)^{-1}|\sigma|^{1 / 2} \operatorname{sign}(\sigma)+K_{e}(\cdot)^{-1} z}_{v_{s}}) \\
& \dot{z}=-k_{2} \operatorname{sign}(\sigma)
\end{aligned}
$$


where the virtual control terms $\delta_{a}$ is the original control law defined in (48)-(49), $v_{s}$ is the added sliding mode (super-twist) term and $B_{f 2}$ is defined in (59). From (66) it follows

$$
\begin{aligned}
& \dot{\sigma}=z-k_{1}|\sigma|^{1 / 2} \operatorname{sign}(\sigma) \\
& \dot{z}=-k_{2} \operatorname{sign}(\sigma)
\end{aligned}
$$

which represents a standard second-order sliding mode formulation. If the scalars

$$
k_{2}>0 \text { and } k_{1}^{2}>4 k_{2}
$$

a second-order sliding motion in which $\dot{\sigma}=\sigma=0$ in finite time can be induced [52, 42].

Remark 4.2

Note that the term $B_{f 2}^{T} \Psi^{-1}(\cdot)$ in the control law in (68) is a control allocation term and the scalar virtual control element (in the brackets on the R.H.S of (68)) is distributed to the four individual elevators (since $B_{f 2}^{T} \in \mathbb{R}^{4}$ ). This control structure is quite different from the first order conventional sliding mode control used in [57]. The use of the super-twisting structure ensures a 2nd order sliding motion with a continuous control signal. This structure also performs better because of the relatively slow sampling rate enforced by the benchmark problem set-up.

\subsection{RECONFIGURE FES}

In this paper, all the simulation results were generated using the RECONFIGURE Functional Engineering Simulator which is a simulation software tool based on the MATLAB/SIMULINK modeling and simulation environment. The software is specifically designed to support the industrial verification and benchmarking of the FDI/FTC algorithm prototypes designed by the partners in the RECONFIGURE project. The FES implements all the benchmark scenarios defined by Airbus for the evaluation of the FDI/FTC designs with traditional Monte Carlo analysis, and provides an interface for a worst-case search tool for implementing advanced optimization-based clearance methods [1].

The FES software architecture is based on SIMPLAT which is a simulation infrastructure designed and developed by DEIMOS Space for the production of Functional Engineering Simulators. The SIMPLAT infrastructure is in turn based on the MATLAB/Simulink modelling and simulation environment and provides all the basic functionalities needed by a FES tool, so that

project-specific elements can be rapidly built on top of it. The SIMPLAT operation largely relies on its XML database, which stores model, scenario and simulation parameters [58].

\subsection{Industrial Limitation and Constraints}

On a large civil aircraft, the computational capability of the Flight Control Computer (FCC) is relatively low, and proven, reliable processors must be used for critical applications. Therefore, it is hard to use advanced processors capable of executing on-line optimisation or even wavelet or Fourier transforms in real time. In RECONFIGURE, to allow the industrial partners to evaluate the computational load of the designs, they have all been coded following the Airbus state of practice for FCC software coding using the SAO (Airbus software, Computer-Assisted Specification) library [59], which contains a set of graphical functional blocks (similar to SIMULINK blocks), allowing 
only a limited set of mathematical operations. Then, an automatic generation tool calculates the computational load and produces the code to be implemented on the FCC. The computational load of the LPV/ISM scheme described in the earlier sections was deemed 'very low' when evaluated by Airbus. This is a key facet of the scheme which is proposed, and results from the fact that the ISM elements are retro-fitted to the existing control architecture.

\subsection{Validation activities and flight conditions}

Table I lists the various validation activities performed for the verification of the Guidance and Control laws during the Monte-Carlo campaign. These validation activities will be used for assessing the robustness and performance of the designs in the presence of actuator faults/failures. The set of manoeuvres involved in most of these validation activities will be discussed later.

Table I. Validation activities

\begin{tabular}{cc}
\hline Validation activities & Verification Objective \\
\hline NZLAW01 & Upper load factor protection \\
\hline NZLAW02 & Lower load factor protection \\
\hline NZLAW03 & Upper load factor protection \\
\hline NZLAW04 & Lower load factor protection \\
\hline NZLAW05 & Homogeneous load factor responses \\
\hline NZLAW06 & Response to vertical gust \\
\hline NZLAW08 & Response to longitudinal tailwind gust \\
\hline AOAPR01 & Response in case of thrust increase \\
\hline AOAPR02 & Response in case of thrust reduction \\
\hline AOAPR03 & Response to wind shear \\
\hline Random pilot input tests (RPIT) & Robustness with respect to random input \\
\hline Vertical speed (VS) control law & Load factor performance in VS mode \\
\hline Flight path angle (FPA) control law & Load factor performance in FPA mode \\
\hline
\end{tabular}

For each validation activity, the proposed scheme will be evaluated at various flight conditions defined in Table. II and III. In these tables, 'MFW' and 'MTOW' denote the maximum flight weight and the maximum take-off weight respectively; and 'VLS', 'ARS' and 'AES' represent the minimum selectable speed, the automatic retraction speed for the high-lift system (slats and flaps) and the automatic extension speed for the high-lift system (slats and flaps), respectively. Also note that in Table. III, the slats/flaps configuration number ( 0 to 4 ) indicates different deflection angles of the slats and flaps ( 0 represents the clean configuration i.e. fully retracted for cruise, and 4 being the full configuration i.e. fully extended used mainly for landing). Numerical values with respect to the various weights and speeds cannot be given in this paper due to industrial restrictions. In this paper, 54 different flight conditions which include 6 different mass cases and 9 different flight points (listed in Table. II and III) will be used for Monte-Carlo evaluation purposes in the presence of faults/failures.

\subsection{Design results}

The values for the scalars $k_{1}$ and $k_{2}$ in (68) are selected to be 47.434 and 90 , respectively. Note: in this RECONFIGURE scenario, an FDI module is assumed to be available [2] so that the faults/failures are assumed to be known, i.e. the matrix $W$ in (66) and (68) is known. The proposed 
Table II. Validated mass cases in the presence of actuator faults/failures

\begin{tabular}{cc}
\hline Gross weight (t) & CG(\%) \\
\hline MFW & Max forward CG \\
\hline MFW & Medium CG \\
\hline MFW & Max aft CG \\
\hline MTOW & Max forward CG \\
\hline MTOW & Medium CG \\
\hline MTOW & Max aft CG \\
\hline
\end{tabular}

Table III. Validated flight points in the presence of actuator faults/failures

\begin{tabular}{ccccc}
\hline VCAS $(\mathrm{kt})$ & Zp(ft) & S/F conf & Landing gear & Phase \\
\hline VLS-5 & Ceiling & 0 & Up & 2 \\
\hline VLS & 30000 & 0 & Up & 2 \\
\hline VLS & 15000 & 0 & Up & 2 \\
\hline (ARS+AES)/2-10 & 7500 & 1 & Up & 2 \\
\hline VLS & 7500 & 1 & Up & 2 \\
\hline VLS & 5000 & 2 & Up & 2 \\
\hline VLS & 2000 & 3 & Up & 2 \\
\hline VLS & 1000 & 3 & Down & 2 \\
\hline VLS & 1000 & 4 & Down & 2 \\
\hline
\end{tabular}

super-twisting LPV/ISM scheme redistributes the control signals to the healthy elevators in order to maintain the nominal $C-N Z$ performance in the face of a fault/failure on one of the elevators despite the large plant-model mismatch caused by the manoeuvres defined in the various validation activities. Note that, due to page limitation, only the FES results associated with the most interesting and worst cases are shown in this section.

\subsection{Validation activities 'NZLAWO6'}

The FTC performance associated with the scenarios 'NZLAW06' have been evaluated at the 54 flight conditions listed in Table. II and III. The objectives of the 'NZLAW06' tests are to evaluate the capability of maintaining an acceptable load factor performance in the presence of actuator faults/failures and vertical and longitudinal wind gusts. Figure 4 shows the vertical gust involved in 'NZLAW06' scenario. During this validation scenario, the AutoThrust is engaged from the beginning of the simulation. As shown in Fig. 7, the switching function remains close to zero. Figure 9 shows the elevator commands which represents one component of $u_{e}$ generated according to (68). It can be seen that the elevator commands, generated by the super-twisting control law are smooth, and satisfy the industrial requirements. Then, the elevator commands in Fig. 9 will be redistributed to the four elevators, one of which is randomly chosen to fail at each flight condition. The control surface deflections associated with four elevators are shown in Fig. 8 where the red lines are associated with the flight conditions in which the elevators are stuck at zero. The FTC $C$ $N Z$ performance in the presence of actuator faults/failures is shown in Fig. 5 and Fig. 6. The absolute value of the difference associated with $n_{z}$ and $q$ performance between the nominal and faulty cases are shown in Fig. 5(a) and Fig. 6(a). The absolute values of the differences associated with $n_{z}$ and $q$ performance between the nominal and the LPV/ISM cases are shown in Fig. 5(b) and Fig. 6(b). It is 
clear that the nominal load factor performance can be retained over a wide range of flight conditions in the presence of actuator faults/failures and wind gusts.

\subsection{Validation activities 'NZLAW02' and 'NZLAW03'}

The evaluation results associated with 'NZLAW02' and 'NZLAW03' are shown in Figs. 10-15. The manoeuvres in these two scenarios involve various pilot pitch stick controller deflections, which are shown in Fig. 10 and Fig. 13. These pilot excitations are aggressive, and are deliberately chosen to activate the load factor and/or velocity protection components inside the RECONFIGURE benchmark. Since these components are 'invisible' for the purpose of design, maintaining nominal performance in the face of the faults/failures becomes a challenging problem. This is another motivation for selecting a sliding mode based approach due to its strong robustness properties $[49,46]$.

Again in the 'NZLAW02' and 'NZLAW03', the AutoThrust is engaged from the beginning of the simulation. Although spikes can be seen corresponding to the step changes in the pilot pitch stick deflections, the switching functions return to close to zero despite sustained aggressive excitations. The absolute value of the difference between the nominal $n_{z}$ performance and the performance in the faulty case (when the ISM scheme is not retro-fitted) are shown in Fig. 11(a) and Fig. 14(a). The absolute values of the differences between the nominal $n_{z}$ performance and the performance in the presence of faults when the LPV/ISM scheme is used are shown in Fig. 11(b) and Fig. 14(b). Clearly, the nominal protected $n_{z}$ performance can be recovered using the ISM scheme. Similarly, from Fig. 12 and Fig. 15, the nominal protected $q$ performance can also be recovered. Since both the nominal $n_{z}$ and $q$ performance can be recovered, the overall nominal $C$-NZ performance has been retained in the presence of actuator faults/failures.

\subsection{Validation activity 'NZLAW05'}

It is important to have homogenous responses across the flight envelope so that the outer-loops can make the assumption of an ideal response to load factor demand. The 'NZLAW05' scenario is used to evaluate the capability of maintaining a homogenous response in the presence of actuator faults/failures. The pilot excitations in Fig. 16 show a sequence of positive and negative stick deflections are involved. Again, the AutoThrust is engaged during the whole simulation. The absolute value of the difference associated with $n_{z}$ and $q$ performance between the nominal and the faulty cases in the absence of the FTC scheme are shown in Fig. 17(a) and Fig. 18(a). The absolute value of the difference associated with $n_{z}$ and $q$ performance between the nominal and the ISM cases are shown in Fig. 17(b) and Fig. 18(b). Clearly from Fig. 17 and 18, nominal $C$-NZ performance can be retained in the presence of actuator faults/failures across various flight conditions.

\subsection{Validation activities 'AOAPRO1'}

In scenario 'AOAPR01', the injected pilot pitch stick deflections are shown in Fig. 19(a). Meanwhile, the throttle is pulled to Maximum Climb Thrust (MCT) as shown in Fig. 19(b). The $n_{z}$ performance in the 'AOAPR01' scenario is shown in Fig. 20, and the $q$ performance is shown in Fig. 21. Figure 20 and Fig. 21 show that the nominal $C$ - $N Z$ performance can be retained in the 
presence of actuator faults/failures and MCT/IDLE throttle commands over a wide range of flight conditions.

\subsection{Validation activity 'AOAPRO3'}

The 'AOAPR03' scenario is shown in Fig. 22 in which both longitudinal and vertical wind is considered. The AutoThrust is engaged during the whole simulation. The absolute values of the differences associated with $n_{z}$ and $q$ performance between the nominal and faulty cases are shown in Fig. 23(a) and Fig. 24(a), respectively. The absolute value of the difference associated with $n_{z}$ and $q$ performance between the nominal and LPV/ISM cases are shown in Fig. 23(b) and Fig. 24(b), respectively. Clearly, nominal $C$-NZ performance can be retained in the presence of actuator faults/failures.

\subsection{Validation activity 'RPIT'}

In 'RPIT', ten random pilot excitations are considered. (One random pilot excitation is generated at one of ten random points in the flight domain). The manoeuvres are shown in Fig. 25 and combine pilot pitch stick deflections, roll stick deflections, pedal deflections and engine thrust inputs. The absolute values of the differences associated with $n_{z}$ and $q$ performance between the nominal and faulty cases are shown in Fig. 26(a) and Fig. 27(a). The absolute value of the difference associated with $n_{z}$ and $q$ performance between the nominal and LPV/ISM cases are shown in Fig. 26(b) and Fig. 27(b). Figure 26 and Fig. 27 show nominal $C$-NZ performance is retained in the presence of actuator faults/failures despite random selected tests.

\subsection{Validation activities for 'VS control law'}

The 'VS control law' scenarios involves the Auto-Pilot mode. Even in Auto-Pilot mode, the dynamics of the load factor law (inner-loop) must be satisfied. The manoeuvres associated with the 'VS control law' are shown in Fig. 28, which contains both the flight path angle and the vertical speed targets, longitudinal and vertical turbulence and vertical wind. It is also clear from Fig. 29 and Fig. 30 that the nominal $C$-NZ performance is retained in the 'VS control law' scenario in the face of actuator faults/failures for a wide range of flight conditions.

\section{CONCLUSIONS}

This paper has developed and applied a novel retro-fitted LPV/ISM scheme to a high-fidelity RECONFIGURE aircraft benchmark model. The scheme aims to maintain nominal closed-loop performance in the presence of actuator faults/failures, despite various validation activities and flight conditions. In order to reduce chattering in the elevator demands, the retro-fitted control inputs are based on a super-twisting sliding mode structure. The industrial FES Monte-Carlo campaign results demonstrate the efficacy of the proposed scheme despite the presence of faults/failures, uncertainties and wide variations of flight conditions and manoeuvres. 


\section{ACKNOWLEDGEMENT}

The work in this paper is supported by European Commission, Seventh Framework Programme, FP7

Transport, Grant/Award Number: 314544.

\section{REFERENCES}

1. Goupil P, Boada-Bauxell J, Marcos A, Rosa P, Kerr M, Dalbies L. An overview of the FP7 RECONFIGURE project: Industrial, scientific and technological objectives. SAFEPROCESS'15, vol. 48, Paris, France, 2015; 976-981.

2. Goupil P, Boada-Bauxell J, Marcos A, Cortet E, Kerr M, Costa H. AIRBUS efforts toward advanced real-time fault diagnosis and fault tolerant control. 19th IFAC World Congress, Cape Town, South Africa, 2014; 3741-3746.

3. Clancy LJ. Aerodynamics. Pitman Publishing Limited, London, 1975.

4. Chen J, Patton RJ. Robust model-based fault diagnosis for dynamic systems. Kluwer academic publishers: Norwell, 1999.

5. Blanke M, Kinnaert M, Lunze J, Staroswiecki M. Introduction to Diagnosis and Fault-Tolerant Control. Springer Berlin Heidelberg: Heidelberg, 2016; 1-35.

6. Ding SX. Model-Based Fault Diagnosis Techniques. Springer: London, 2013.

7. Yu X, Jiang J. A survey of fault-tolerant controllers based on safety-related issues. Annual Reviews in Control 2015; 39:46-57.

8. Zhang Y, Jiang J. Bibliographical review on reconfigurable fault-tolerant control systems. Annual Reviews in Control 2008; 32:229 - 252 .

9. Benini M, Castaldi P, Simani S. Fault Diagnosis for Aircraft System Models: An Introduction from Fault Detection to Fault Tolerance. VDM Verlag Dr. Muller: Saarbrcken, 2009.

10. Edwards C, Lombaerts T, Smaili H. Fault tolerant flight control: A benchmark challenge. Springer: Berlin Heidelberg, 2010.

11. Goupil P. AIRBUS state of the art and practices on FDI and FTC in flight control system. Control Eng. Pract. 2011; 19:524 - 539 .

12. Zolghadri A, Henry D, Cieslak J, Efimov D, Goupil P. Fault Diagnosis and Fault-Tolerant Control and Guidance for Aerospace Vehicles, from theory to application. Springer, Series: Advances in Industrial Control: London, 2014.

13. Alwi H, Edwards C, Tan CP. Fault Detection and Fault-Tolerant Control Using Sliding Modes. Springer: London, 2011.

14. Durham W, Bordignon KA, Beck R. Aircraft Control Allocation. John Wiley \& Sons: Chichester, 2017.

15. Johansen TA, Fossen TI. Control allocation - A survey. Automatica 2013; 49:1087-1103.

16. Zhang Y, Suresh VS, Jiang B, Theilliol D. Reconfigurable Control Allocation against Aircraft Control Effector Failures. 16th IEEE International Conference on Control Applications, Singapore, 2007; 1197-1202.

17. Smaili MH, Breeman J, Lombaerts TJJ, Mulder JA, Chu QP, Stroosma O. Intelligent Flight Control Systems Evaluation for Loss-of-Control Recovery and Prevention. AIAA Journal of Guidance Control and Dynamics 2017; 40:890-904.

18. Lombaerts TJJ, Chu QP, Mulder JA, Joosten DA. Modular flight control reconfiguration design and simulation ; .

19. Nayebpanah N, Rodrigues L, Zhang Y. Fault tolerant control for partial loss of control authority in aircraft using piecewise affine slab models. J. Franklin Inst. 2013; 350:2494 - 2508.

20. Maciejowski JM, Hartley EN, Siaulys K. A longitudinal flight control law to accommodate sensor loss in the RECONFIGURE benchmark. Annual Reviews of Control 2016; 42:212-223.

21. de Almeida FA, Leissling D. Fault-Tolerant Model Predictive Control with Flight-Test Results. AIAA Journal of Guidance Control and Dynamics 2010; 33:363-375.

22. Sun LG, de Visser CC, Chu QP, Mulder JA. Joint Sensor Based Backstepping for Fault-Tolerant Flight Control. AIAA Journal of Guidance Control and Dynamics 2015; 38:62-75.

23. Shen Q, B Jiang B, Cocquempot V. Adaptive fault-tolerant backstepping control against actuator gain faults and its applications to an aircraft longitudinal motion dynamics. Int. J. Robust Nonlinear Control 2013; 23:1753-1779.

24. Lu P, van Kampen EJ, de Visser C, Chu Q. Aircraft fault-tolerant trajectory control using Incremental Nonlinear Dynamic Inversion. Control Eng. Pract. 2016; 57:126-141.

25. Fan W, Liu HHT, Kwong RHS. Gain-Scheduling Control of Flexible Aircraft with Actuator Saturation and Stuck Faults. AIAA Journal of Guidance Control and Dynamics 2017; 40:510-520.

26. Jacobson CA, Nett CN. An integrated approach to controls and diagnostics using the four parameter controller. IEEE Control Syst. 1991; 11:22-29. 
27. Chen L, Patton R, Goupil P. Robust fault estimation using an LPV reference model: ADDSAFE benchmark case study. Control Eng. Pract. 2016; 49:194-203.

28. Sato M, Peaucelle D. Gain-scheduled output-feedback controllers using inexact scheduling parameters for continuous-time LPV systems. Automatica 2013; 49:1019-1025.

29. Yu X, Jiang J. Fault-Tolerant Flight Control System Design Against Control Surface Impairments. IEEE Trans Aerosp Electron Syst 2012; 48:1031-1051.

30. Vanek B, Edelmayer A, Szabo Z, Bokor J. Bridging the gap between theory and practice in LPV fault detection for flight control actuators. Control Eng. Pract. 2014; 31:171-182.

31. Cieslak J, Henry D, Zolghadri A. Fault tolerant flight control: from theory to piloted flight simulator experiments. IET Control Theory Appl. 2010; 4:1451-1464.

32. Rotondo D, Nejjari F, Puig V, Blesa J. Model reference FTC for LPV systems using virtual actuator and setmembership fault estimation. Int. J. Robust Nonlinear Control 2015; 25:753-760.

33. Gregory I, Xargay E, Cao C, Hovakimyan N. Flight test of L1 adaptive controller on the NASA AirSTAR flight test vehicle. AIAA GNC, Toronto, Ontario, Canada, 2010, doi:10.2514/6.2010-8015.

34. Gao G, Wang J, Wang X. Adaptive fault-tolerant control for feedback linearizable systems with an aircraft application. Int. J. Robust Nonlinear Control 2015; 25:1301-1326.

35. Boskovic JD, Mehra RK. A Decentralized Fault-Tolerant Control System for Accommodation of Failures in HigherOrder Flight Control Actuators. IEEE Trans. Control Syst. Technol. 2010; 18:1103-1115.

36. Jin XZ, Yang GH, Chang XH. Robust H-infinity and adaptive tracking control against actuator faults with a linearised aircraft application. Int. J. Syst. Sci. 2013; 44:151-165.

37. Yu X, Zhang Y, Liu Z. Fault-Tolerant Flight Control Design with Explicit Consideration of Reconfiguration Transients. AIAA Journal of Guidance Control and Dynamics 2016; 39:556-563.

38. Yu X, Liu Z, Zhang Y. Fault-Tolerant Flight Control Design With Finite-Time Adaptation Under Actuator Stuck Failures. IEEE Trans. Control Syst. Technol. 2017; 25:1431-1440.

39. Chakravarty A, Mahanta C. Actuator fault-tolerant control (FTC) design with post-fault transient improvement for application to aircraft control. Int. J. Robust Nonlinear Control 2016; 26:2049-2074.

40. Efimov D, Fridman L, Raissi T, Zolghadri A, Seydou R. Interval estimation for LPV systems applying high order sliding mode techniques. Automatica 2012; 48:2365-2371.

41. Wang T, Xie W, Zhang Y. Sliding mode fault tolerant control dealing with modeling uncertainties and actuator faults. ISA Transactions 2012; 51:386-392.

42. Shtessel Y, Edwards C, Fridman L, Levant A. Sliding Mode Control and Observation. Birkhauser: Basel, 2013.

43. Castanos F, Fridman L. Analysis and design of integral sliding manifolds for systems with unmatched perturbations. IEEE Trans. Autom. Control. 2006; 51:853-855.

44. Hamayun MT, Edwards C, Alwi H. Augmentation scheme for fault-tolerant control using integral sliding modes. IEEE Trans. Control Syst. Technol. 2014; 22:307-313.

45. Levant A. Robust exact differentiation via sliding mode technique. Automatica 1998; 34:379-384.

46. Hamayun MT, Edwards C, Alwi H. Fault tolerant control schemes using integral sliding modes. Springer: Cham, 2016.

47. Zhang K, Jiang B, Yan XG, Mao Z. Adaptive robust fault-tolerant control for linear MIMO systems with unmatched uncertainties. Int. J. Control 2017; 90:2253-2269.

48. Alwi H, Edwards C. Fault detection and fault-tolerant control of a civil aircraft using a sliding-mode-based scheme. IEEE Trans. Control Syst. Technol. 2008; 16:499-510.

49. Utkin V. Sliding modes in control and optimization. Springer: Heidelberg, 1992.

50. Harkegard O, Glad S. Resolving actuator redundancy - optimal vs. control allocation. Automatica 2005; 41:137144.

51. Deimling K. Multivalued differential equations. Berlin, Germany: Walter De Gruyer, 1992.

52. Nagesh I, Edwards C. A multivariable super-twisting sliding mode approach. Automatica 2014; 50:984-988.

53. Gonzalez T, Moreno J, Fridman L. Variable gain super-twisting sliding mode control. IEEE Trans. Autom. Control. 2012; 57:2100-2105.

54. Edwards C, Shtessel Y. Adaptive dual-layer super-twisting control and observation. Int. J. Control 2016; 89:17591766.

55. Edwards C, Shtessel Y. Adaptive continuous higher order sliding mode control. Automatica 2016; 65:183 - 190.

56. Hecker S, Pififer H. Affine LPV-modeling for the ADDSAFE benchmark. Control Eng. Pract. 2014; 31:126-134.

57. Chen L, Alwi H, Edwards C. Development and application of an LPV fault tolerant integral sliding mode control allocation scheme for the RECONFIGURE benchmark model. SAFEPROCESS'15, vol. 48, Paris, France, 2015; 994-999. 
58. Fernández V, Montano J, Recupero C, Kerr M, Rosa P, Boada-Bauxell J, Dalbies L. A tool for industrial verification and benchmarking of FDD/FTC designs. SAFEPROCESS'15, vol. 48, Paris, France, 2015; 1006-1011.

59. Goupil P, Dayre R, Brot P. From theory to flight tests: Airbus Flight Control System TRL5 achievements. 19th IFAC World Congress, vol. 47, Cape Town, South Africa, 2014; 3497-3502. 


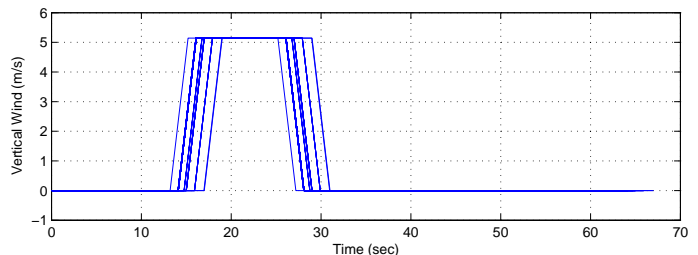

Figure 4. Vertical wind in 'NZLAW06'

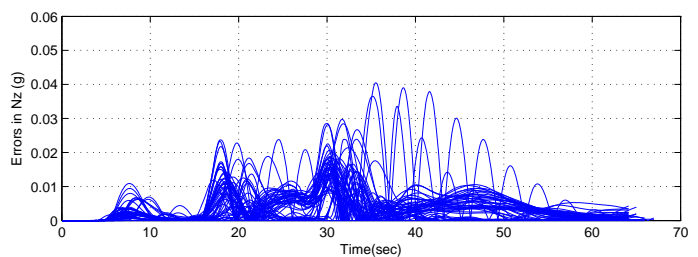

(a) Errors between the nominal and the faulty cases

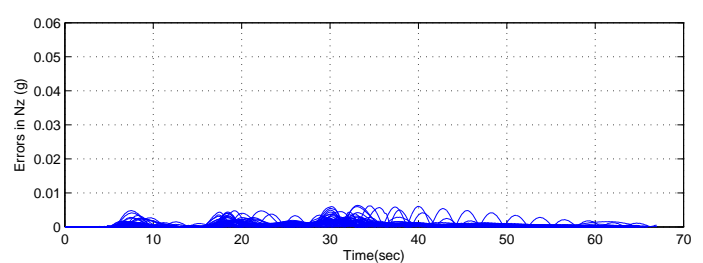

(b) Errors between the nominal and the ISM cases

Figure 5. $n_{z}$ performance in 'NZLAW06'

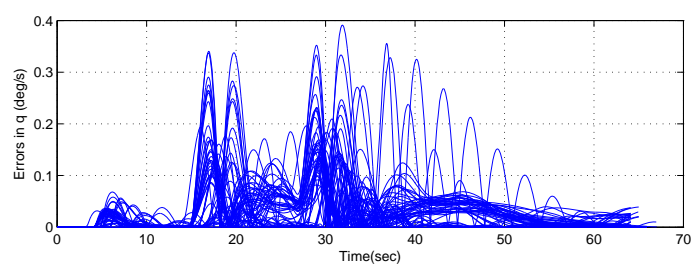

(a) Errors between the nominal and the faulty cases

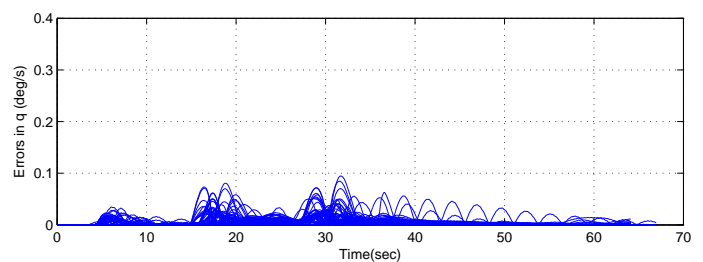

(b) Errors between the nominal and the ISM cases

Figure 6. $q$ performance in 'NZLAW06'

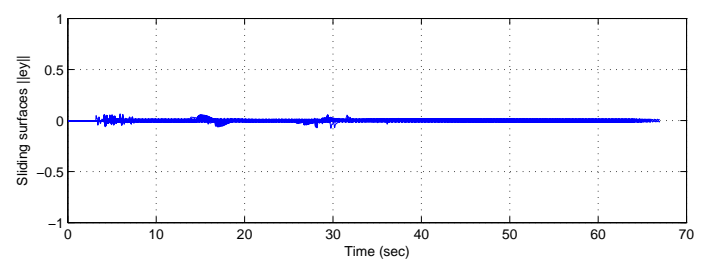

Figure 7. Switching functions in 'NZLAW06'

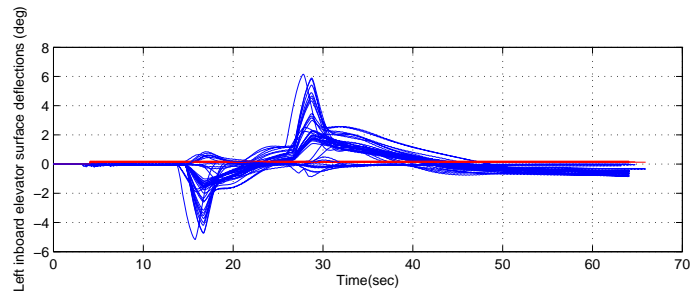

(a) Left inboard elevator surface deflections

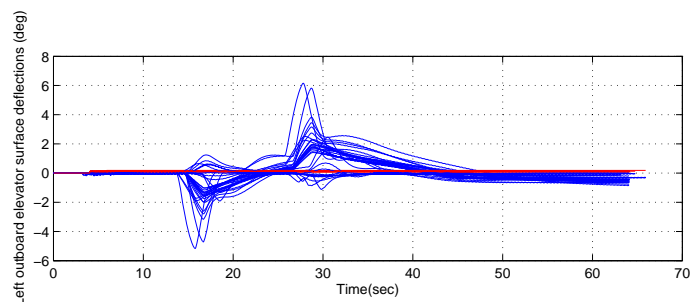

(b) Left outboard elevator surface deflections

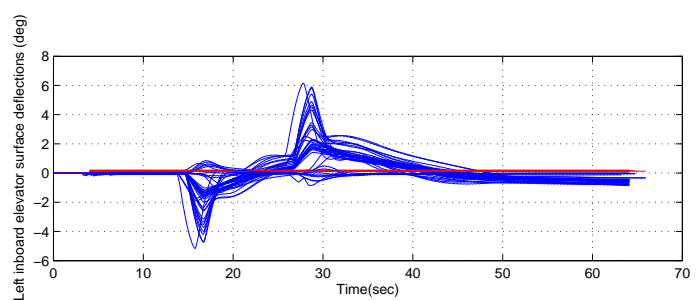

(c) Right inboard elevator surface deflections

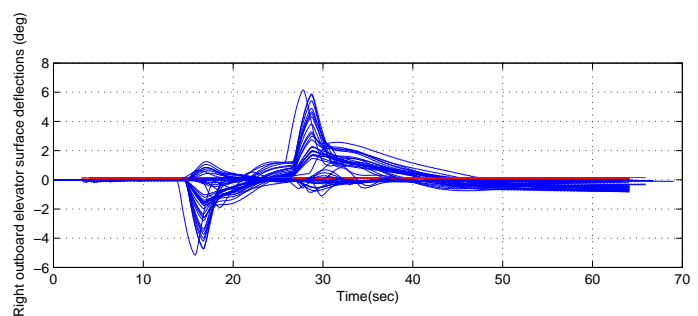

(d) Right inboard elevator surface deflections

Figure 8. Control surface deflections in 'NZLAW06' 

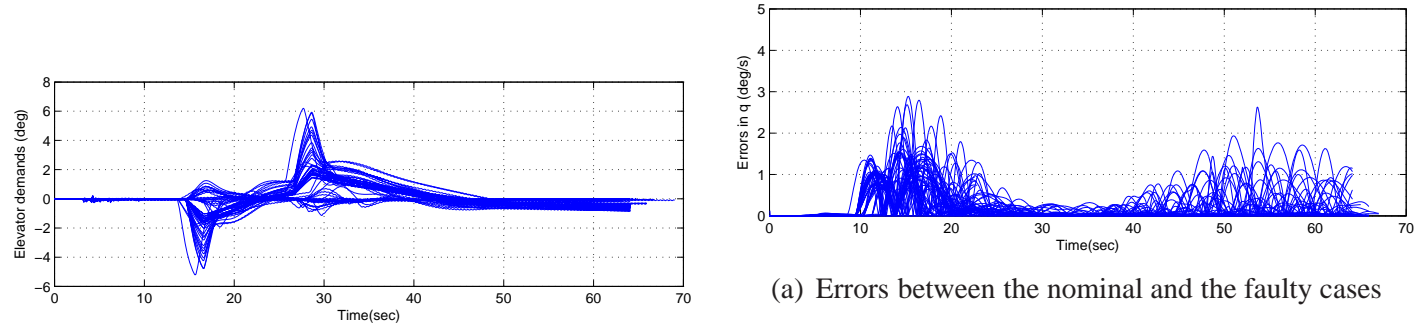

(a) Errors between the nominal and the faulty cases

Figure 9. Elevator deflection orders in 'NZLAW06'

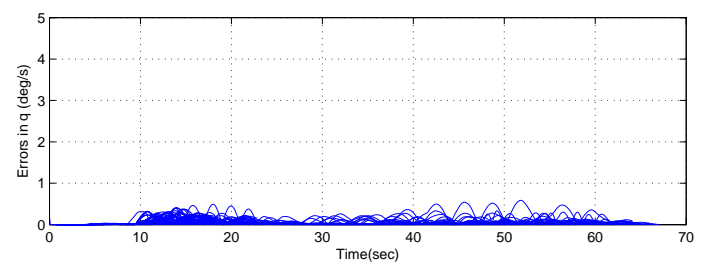

(b) Errors between the nominal and the ISM cases

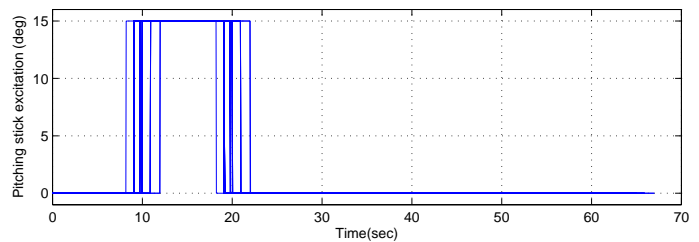

Figure 10. Pitching stick excitations in 'NZLAW02'

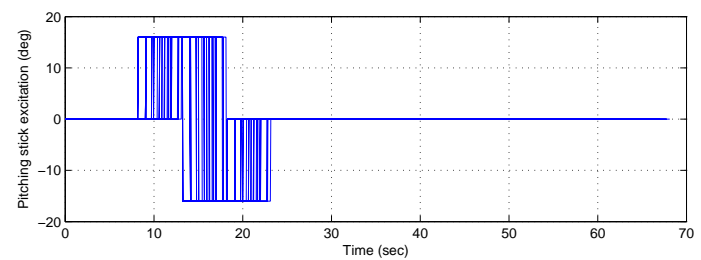

Figure 13. Pitching stick excitations in 'NZLAW03'

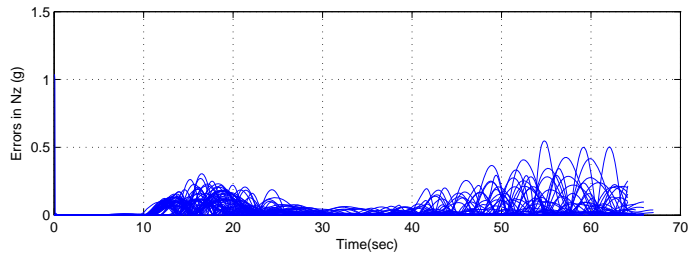

(a) Errors between the nominal and the faulty cases

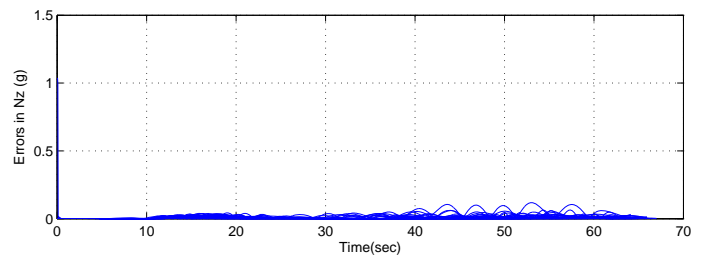

(b) Errors between the nominal and the ISM cases

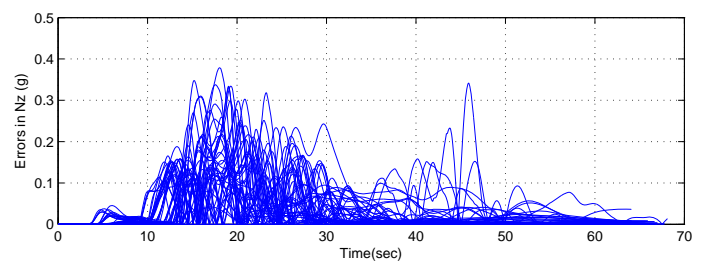

(a) Errors between the nominal and the faulty cases

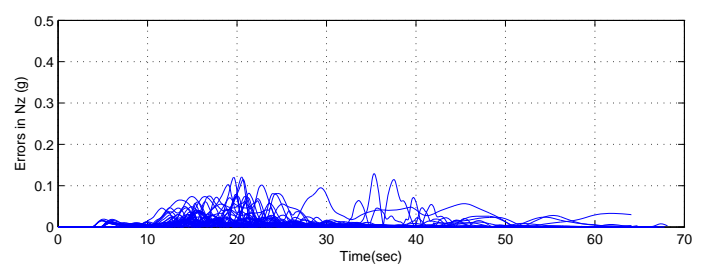

(b) Errors between the nominal and the ISM cases

Figure 14. $n_{z}$ performance in 'NZLAW03' 


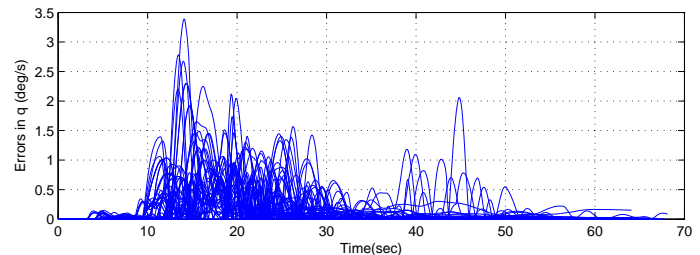

(a) Errors between the nominal and the faulty cases

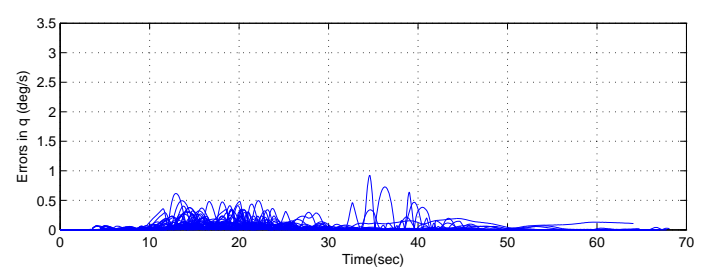

(b) Errors between the nominal and the ISM cases

Figure 15. $q$ performance in 'NZLAW03'

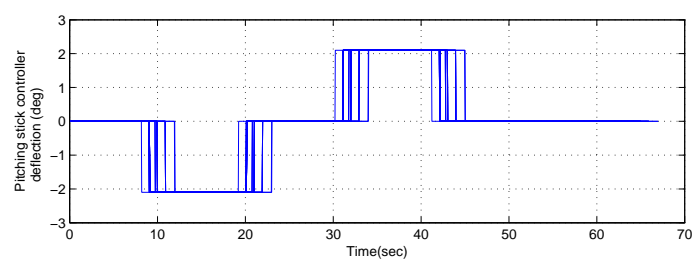

Figure 16. Pitching stick excitations in 'NZLAW05'

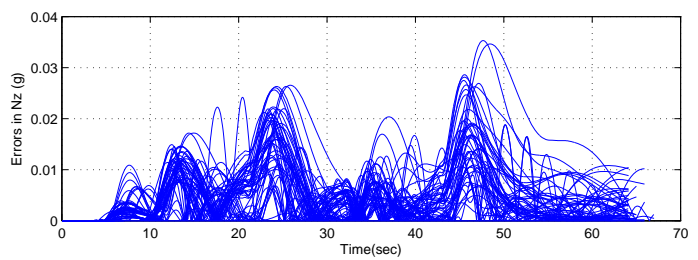

(a) Errors between the nominal and the faulty cases

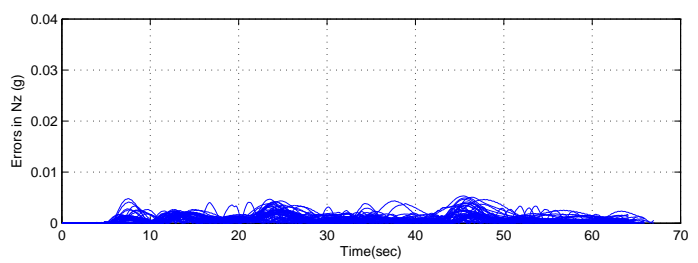

(b) Errors between the nominal and the ISM cases

Figure 17. $n_{z}$ performance in 'NZLAW05'

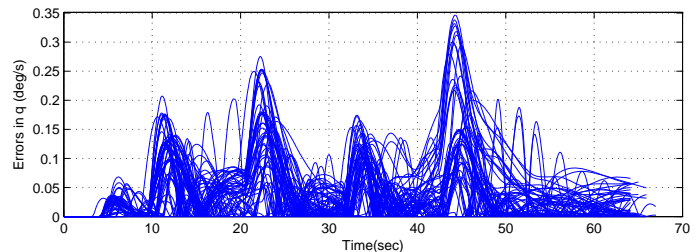

(a) Errors between the nominal and the faulty cases

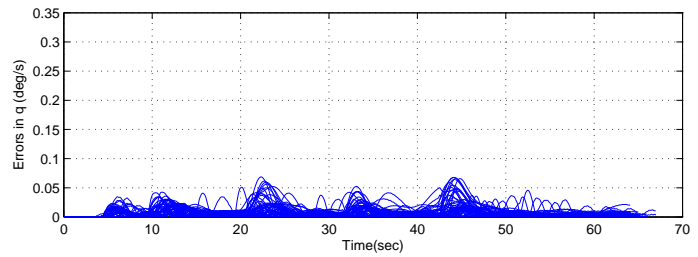

(b) Errors between the nominal and the ISM cases

Figure 18. $q$ performance in 'NZLAW05'

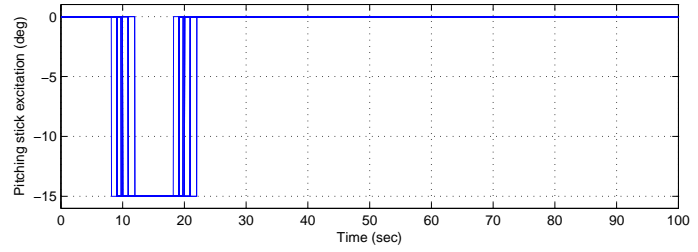

(a) Pitching stick excitations

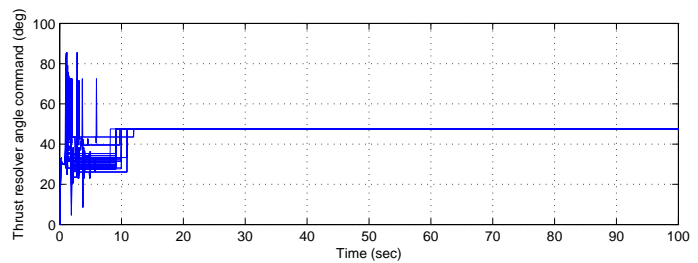

(b) Thrust resolver angle commands

Figure 19. Manoeuvres involved in 'AOA01' 


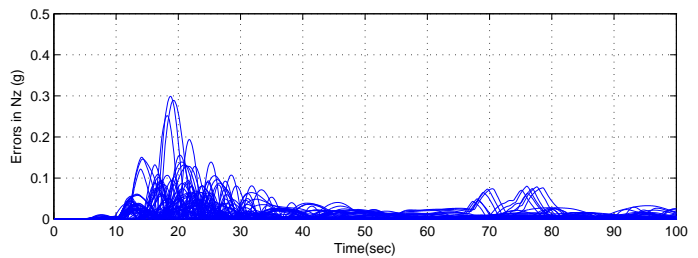

(a) Errors between the nominal and the faulty cases

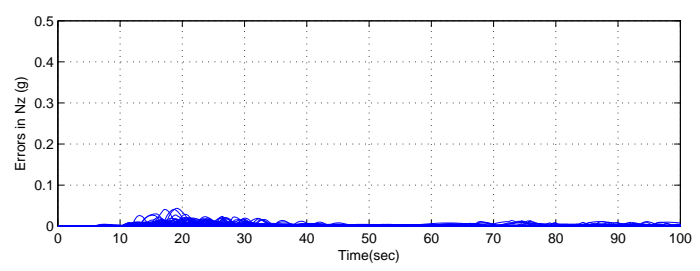

(b) Errors between the nominal and the ISM cases

Figure 20. $n_{z}$ performance in 'AOA01'

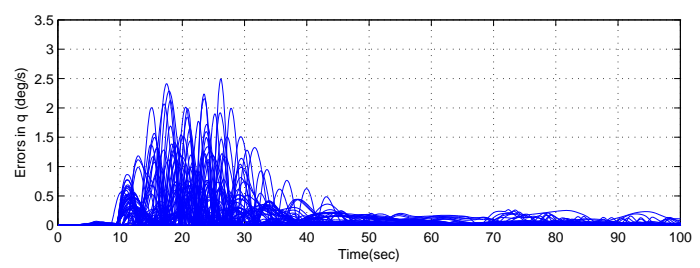

(a) Errors between the nominal and the faulty cases

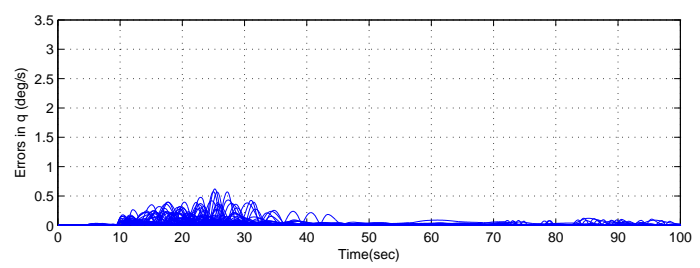

(b) Errors between the nominal and the ISM cases

Figure 21. $q$ performance in 'AOA01'

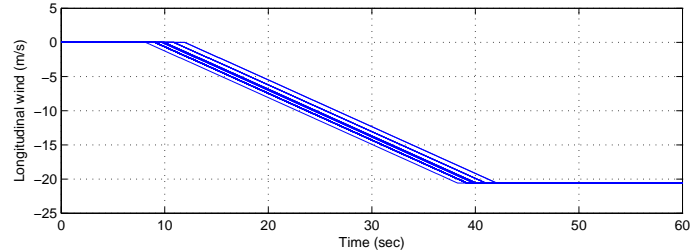

(a) Longitudinal wind

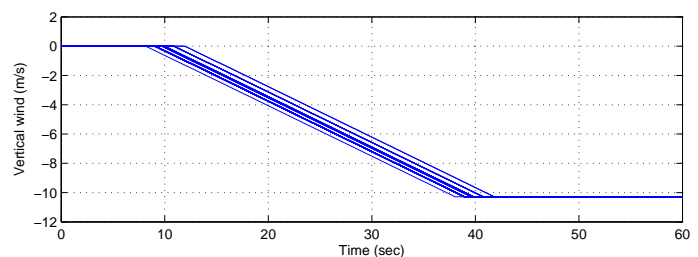

(b) Vertical wind

Figure 22. Manoeuvres involved in 'AOA03'

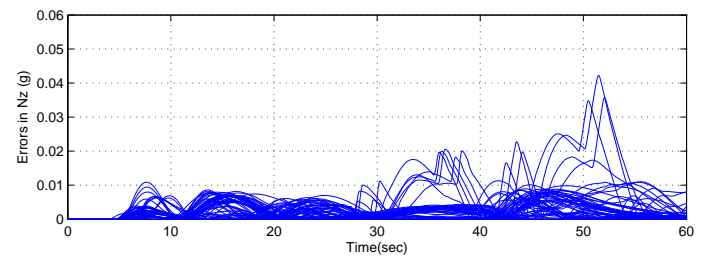

(a) Errors between the nominal and the faulty cases

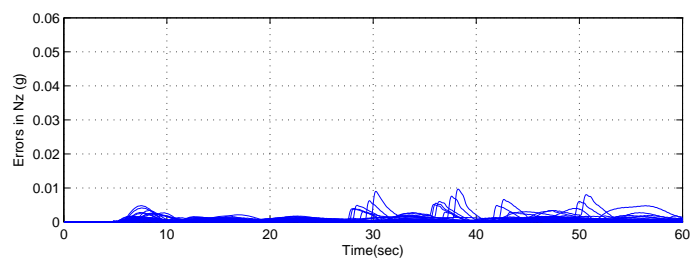

(b) Errors between the nominal and the ISM cases

Figure 23. $n_{z}$ performance in 'AOA03' 


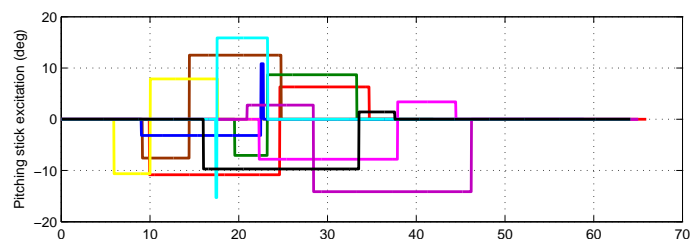

(a) Pitching stick controller deflections

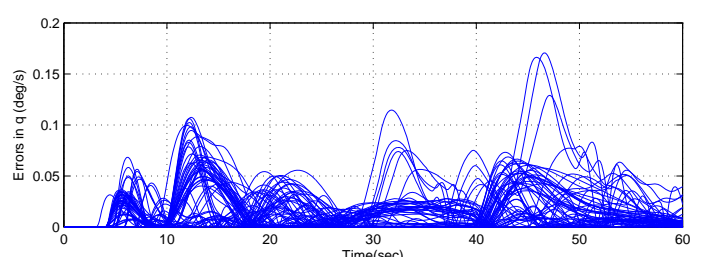

(a) Errors between the nominal and the faulty cases

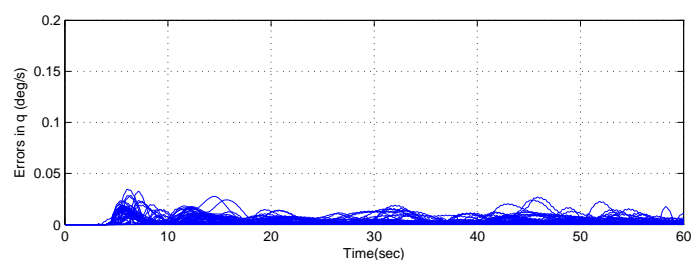

(b) Errors between the nominal and the ISM cases

Figure 24. $q$ performance in 'AOA03'

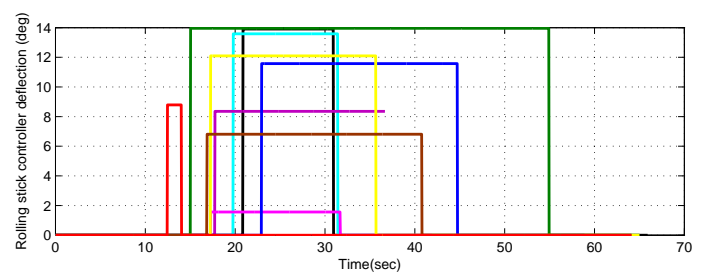

(b) Rolling stick controller deflections

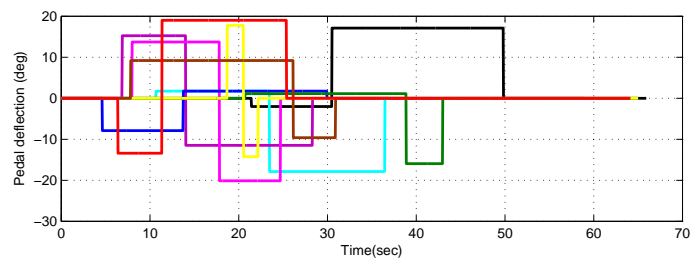

(c) Pedal deflections

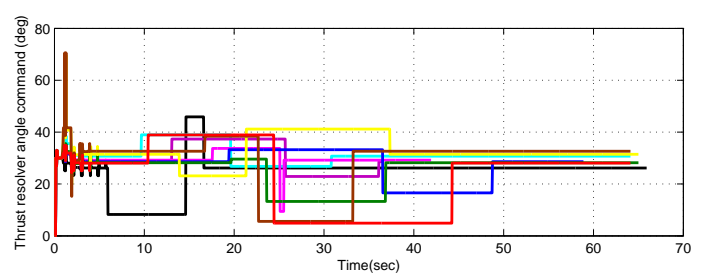

(d) Thrust resolver angle commands

Figure 25. Manoeuvres involved in 'RPIT' 


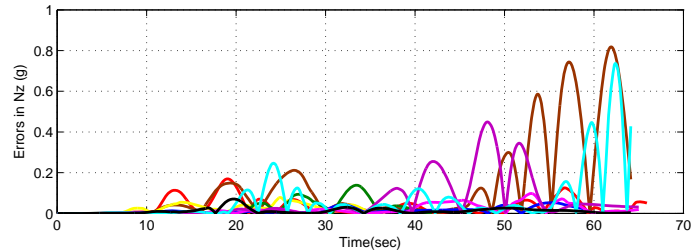

(a) Errors between the nominal and the faulty cases

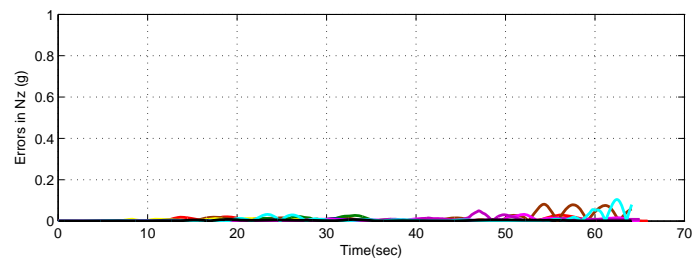

(b) Errors between the nominal and the ISM cases

Figure 26. $n_{z}$ performance in 'RPIT'

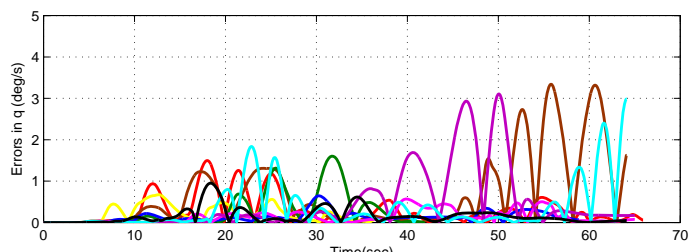

(a) Errors between the nominal and the faulty cases

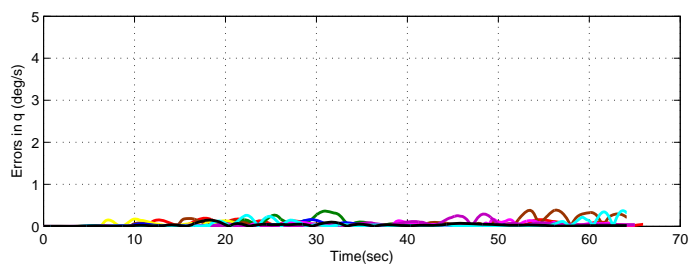

(b) Errors between the nominal and the ISM cases

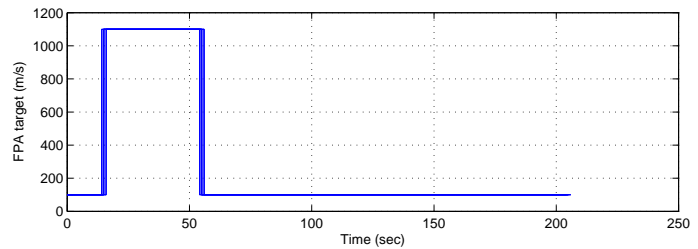

(a) FPA targets

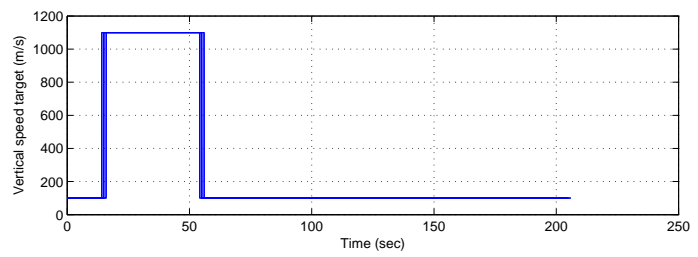

(b) VS targets

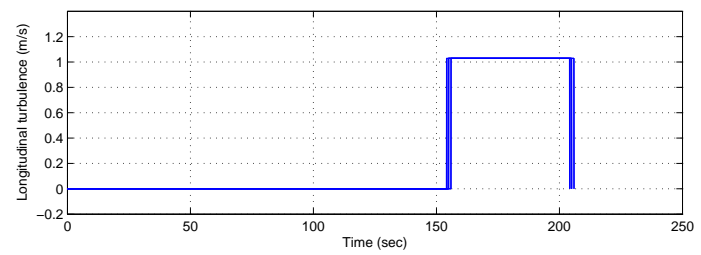

(c) Longitudinal turbulence

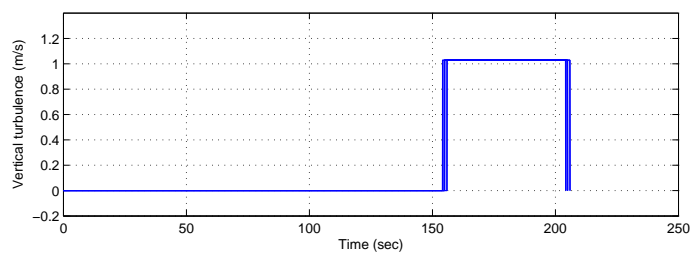

(d) Vertical turbulence

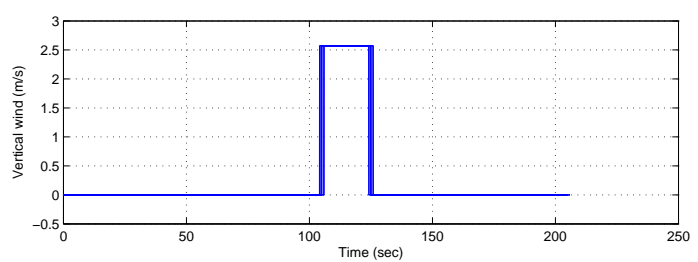

(e) Vertical wind

Figure 28. Manoeuvres involved in 'VS control'

Figure 27. $q$ performance in 'RPIT' 


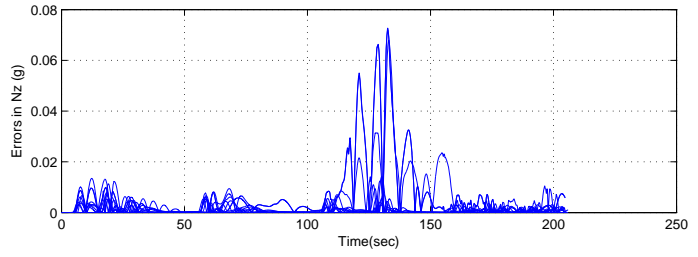

(a) Errors between the nominal and the faulty cases

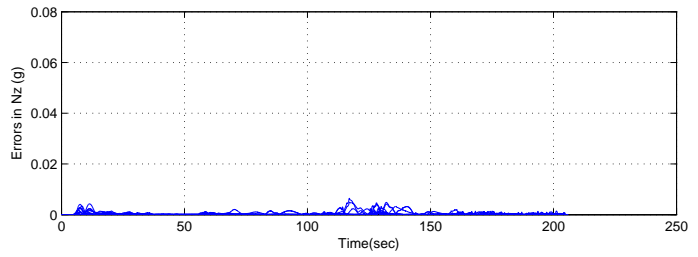

(b) Errors between the nominal and the ISM cases

Figure 29. $n_{z}$ performance in 'VS control'

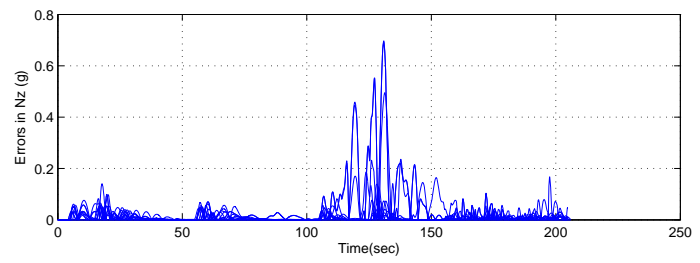

(a) Errors between the nominal and the faulty cases

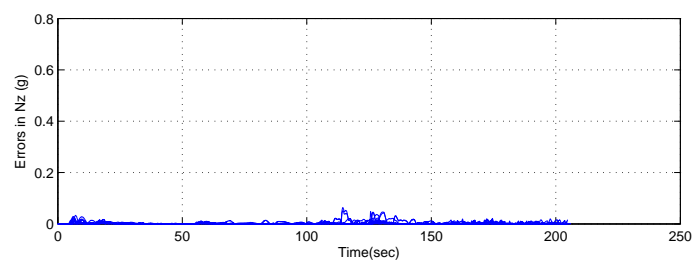

(b) Errors between the nominal and the ISM cases

Figure 30. $q$ performance in 'VS control' 